\title{
Search for the standard model Higgs boson in the $\mathrm{H} \rightarrow \mathrm{ZZ} \rightarrow \ell^{+} \ell^{-} \boldsymbol{\tau}^{+} \tau^{-}$decay channel in pp collisions at $\sqrt{s}=7 \mathrm{TeV}$
}

\section{The CMS collaboration}

ABstract: A search is reported for the standard model Higgs boson in the $\mathrm{H} \rightarrow \mathrm{ZZ} \rightarrow$ $\ell^{+} \ell^{-} \tau^{+} \tau^{-}$decay mode, where $\ell=\mu$ or e, in proton-proton collisions at $\sqrt{s}=7 \mathrm{TeV}$, corresponding to an integrated luminosity of $4.7 \mathrm{fb}^{-1}$ collected with the CMS detector at the LHC. No evidence is found for a significant deviation from the background expectation. An upper limit four to twelve times larger than the predicted value is set at $95 \%$ confidence level for the product of the standard model Higgs boson production cross section and decay branching fraction in the mass range $190<m_{\mathrm{H}}<600 \mathrm{GeV}$.

KEYwORDS: Hadron-Hadron Scattering 


\section{Contents}

1 Introduction 1

2 CMS detector $\quad 2$

3 Event selection and Monte Carlo samples 2

4 Background estimates and systematic uncertainties 4

5 Results 5

$\begin{array}{lll}6 & \text { Summary } & 7\end{array}$

$\begin{array}{ll}\text { The CMS collaboration } & 13\end{array}$

\section{Introduction}

The search for the standard model (SM) [1-3] Higgs boson is one of the main goals of the Large Hadron Collider (LHC) physics programme. The discovery of the SM Higgs boson would shed light on the spontaneous electroweak symmetry breaking mechanism [49]. To date, experimental searches for this particle have yielded null results. Limits at 95\% confidence level (CL) on its mass have been placed by experiments at LEP, $m_{\mathrm{H}}>$ $114.4 \mathrm{GeV}$ [10], the Tevatron, $m_{\mathrm{H}} \notin(162-166) \mathrm{GeV}$ [11], and ATLAS, $m_{\mathrm{H}} \notin(145-206)$, (214-224), (340-450) GeV [12-14]. Precision electroweak measurements, not taking into account the results from direct searches, indirectly constrain the SM Higgs boson mass to be less than $158 \mathrm{GeV}$ [15].

This letter presents a search for the SM Higgs boson in the decay mode $\mathrm{H} \rightarrow \mathrm{ZZ} \rightarrow$ $\ell^{+} \ell^{-} \tau^{+} \tau^{-}$, where $\ell$ is either $\mu$ or e. One $\mathrm{Z}$ is required to decay either into $\mu^{+} \mu^{-}$or $\mathrm{e}^{+} \mathrm{e}^{-}$, and the second $\mathrm{Z}$ into $\tau^{+} \tau^{-}$in four possible final states: $\tau_{\mathrm{h}} \tau_{\mathrm{h}}, \tau_{\mu} \tau_{\mathrm{h}}, \tau_{\mathrm{e}} \tau_{\mathrm{h}}$, and $\tau_{\mathrm{e}} \tau_{\mu}$, where $\tau_{\mathrm{h}}$ represents a $\tau$ decaying hadronically, and $\tau_{\mu}$, and $\tau_{\mathrm{e}}$ indicate taus decaying into muons and electrons respectively. The present measurement complements the search in the $\mathrm{H} \rightarrow \mathrm{ZZ} \rightarrow 4 \ell$ channel [16]. The presence of four leptons in the final state provides a clean signature with only a small contribution from background processes. The major irreducible background contribution is non-resonant $\mathrm{ZZ}$ production. The most important reducible background contributions are $\mathrm{Z}$ and $\mathrm{WZ}$ production in association with jets, and $\mathrm{t} \overline{\mathrm{t}}$ production. The final states $\tau \tau \rightarrow \tau_{\mu} \tau_{\mu}, \tau_{\mathrm{e}} \tau_{\mathrm{e}}$ are not considered, as they are accounted for in the $\mathrm{H} \rightarrow \mathrm{ZZ} \rightarrow 4 \ell$ Higgs search [16]. The cross sections for the Higgs boson production mechanisms and decay branching fractions, together with their uncertainties, are taken from ref. [17] and are derived from refs. [18-37]. 
The analysis is based on data from proton-proton collisions at $\sqrt{s}=7 \mathrm{TeV}$, corresponding to an integrated luminosity of $4.7 \mathrm{fb}^{-1}$ collected with the Compact Muon Solenoid (CMS) detector at the LHC in 2011. This is the first Higgs boson search performed in the $\mathrm{H} \rightarrow \mathrm{ZZ} \rightarrow \ell^{+} \ell^{-} \tau^{+} \tau^{-}$channel.

\section{CMS detector}

A detailed description of the CMS detector can be found elsewhere [38]. The central feature of the CMS apparatus is a superconducting solenoid, of $6 \mathrm{~m}$ internal diameter, providing a magnetic field of $3.8 \mathrm{~T}$. Within the field volume are the silicon pixel and strip tracker, the crystal electromagnetic calorimeter (ECAL), and the brass/scintillator hadron calorimeter. Muons are measured in gas-ionization detectors embedded in the steel return yoke.

CMS uses a right-handed coordinate system, with the origin at the nominal interaction point, the $x$-axis pointing to the centre of the LHC ring, the $y$-axis pointing up (perpendicular to the LHC plane), and the $z$-axis along the counterclockwise-beam direction. The polar angle, $\theta$, is measured from the positive $z$-axis and the azimuthal angle, $\phi$, is measured in the $x-y$ plane. Variables used in this analysis are the pseudorapidity, $\eta=-\ln [\tan (\theta / 2)]$, and the transverse momentum, $p_{\mathrm{T}}=\sqrt{p_{x}^{2}+p_{y}^{2}}$.

The ECAL is designed to have both excellent energy resolution and high granularity, properties that are crucial for reconstructing electrons and photons produced in $\tau$-lepton decays. The ECAL is constructed with projective lead tungstate crystals that provide coverage in pseudorapidity $|\eta|<1.479$ in a barrel region and $1.479<|\eta|<3.0$ in two endcap regions (EE). A preshower detector consisting of two planes of silicon sensors interleaved with a total of $3 X_{0}$ of lead is located in front of the EE. The energy resolution is $3 \%$ or better for the range of electron energies relevant for this analysis.

The inner tracker measures charged particle tracks within the range $|\eta|<2.5$. It consists of 1440 silicon pixel and 15148 silicon strip detector modules, and provides an impact parameter resolution of $\sim 15 \mu \mathrm{m}$ and a transverse momentum resolution of about $1.5 \%$ for $100 \mathrm{GeV}$ particles. The reconstructed tracks are used to measure the location of interaction vertices. The spatial resolution of the reconstruction is $\approx 25 \mu \mathrm{m}$ for vertices with more than 30 associated tracks [39].

The muon barrel region is covered by drift tubes, and the endcap regions by cathode strip chambers. In both regions, resistive plate chambers provide additional coordinate and timing information. Muons are reconstructed in the range $|\eta|<2.4$, with a typical $p_{\mathrm{T}}$ resolution of $\approx 1 \%$ for $p_{\mathrm{T}} \approx 40 \mathrm{GeV}$.

\section{Event selection and Monte Carlo samples}

At the trigger level, the selected events are required to have at least two muons, one with $p_{\mathrm{T}}>13 \mathrm{GeV}\left(p_{\mathrm{T}}>17 \mathrm{GeV}\right.$ for the end of the data-taking period when the instantaneous luminosity was highest) and the other with $p_{\mathrm{T}}>8 \mathrm{GeV}$, or at least two electrons, one with $p_{\mathrm{T}}>17 \mathrm{GeV}$ and the other with $p_{\mathrm{T}}>8 \mathrm{GeV}$. 
Algorithms for identifying muons and electrons, collectively referred to as leptons, are based on the tracker, the muon systems and the calorimeters [40,41]. Since the ZZ final state is expected to have only a small contribution from background processes, the algorithms are tuned to maximize the lepton-reconstruction efficiency, resulting in an increased lepton-misidentification rate. A particle flow ( $\mathrm{PF}$ ) technique [42] is used to form leptonisolation quantities and is also used for $\tau_{\mathrm{h}}$ reconstruction. In the PF approach, information from all subdetectors is combined to reconstruct and identify particles produced in the collision. The particles are classified into mutually exclusive categories: charged hadrons, photons, neutral hadrons, muons, and electrons. These particles are used to reconstruct $\tau_{\mathrm{h}}$ with the "hadron plus strip" (HPS) algorithm [43] that is designed to optimize the performance of $\tau_{\mathrm{h}}$ identification and reconstruction by considering specific $\tau_{\mathrm{h}}$ decay modes. The neutrinos produced in all $\tau$ decays escape detection and are ignored in the $\tau_{\mathrm{h}}$ reconstruction. The algorithm provides high $\tau_{\mathrm{h}}$ identification efficiency, approximately $50 \%$ for the range of $\tau_{\mathrm{h}}$ energies relevant for this analysis, while keeping the misidentification rate for jets at the level of $\approx 1 \%$, that is factor of three to four times lower with respect to other available algorithms [44].

Events are required to have at least one $\mathrm{Z} \rightarrow \ell^{+} \ell^{-}$candidate, denoted by $\mathrm{Z}_{1}$, with the leptons of opposite charge, one with $p_{\mathrm{T}}>20 \mathrm{GeV}$ and another with $p_{\mathrm{T}}>10 \mathrm{GeV}$, and with $|\eta|<2.4$ for the muons and $|\eta|<2.5$ for the electrons. Both leptons are required to have a combined $\mathrm{PF}$ relative isolation $I_{\mathrm{rel}}^{\mathrm{PF}}<0.25$, which is defined as:

$$
I_{\text {rel }}^{\mathrm{PF}}=\left(p_{\mathrm{T}}^{\text {charged }}+\max \left(E_{\mathrm{T}}^{\gamma}+E_{\mathrm{T}}^{\text {neutral }}-0.5 \times p_{\mathrm{T}}^{\mathrm{PU}}, 0\right)\right) / p_{\mathrm{T}}^{\ell},
$$

where $p_{\mathrm{T}}^{\text {charged }}$ is the scalar sum of the charged hadrons $p_{\mathrm{T}}$, and $E_{\mathrm{T}}^{\gamma}$ and $E_{\mathrm{T}}^{\text {neutral }}$ correspond, respectively, to the sum of the transverse energies of the photons and neutral hadrons, all measured in the isolation cone of $\Delta R<0.4$ around the lepton direction, where $\Delta R=\sqrt{(\Delta \eta)^{2}+(\Delta \phi)^{2}}$. The contribution from neutrals is corrected for the effect of overlapping pp interactions. The value of the correction is estimated by scaling the sum of the $p_{\mathrm{T}}$ of all charged particles not associated with the vertex in the isolation cone $\left(p_{\mathrm{T}}^{\mathrm{PU}}\right)$ by a factor 0.5 , which is approximately the ratio of neutral to charged hadron yields in hadronization processes. The visible invariant mass of the reconstructed $\mathrm{Z}_{1}$ is required to be $60<m_{\ell \ell}<120 \mathrm{GeV}$.

For the second $\mathrm{Z}$, denoted by $\mathrm{Z}_{2}$, the selection requirements depend on the final state. If the final state is $\tau_{\mu} \tau_{\mathrm{e}}$, the lepton $p_{\mathrm{T}}$ values are required to exceed $10 \mathrm{GeV}$. The remaining criteria are identical to those for $\mathrm{Z}_{1}$. Since $\tau_{\mathrm{h}} \mathrm{s}$ have much larger misidentification rates than the other leptons, the isolation requirement based on $I_{\text {rel }}^{\mathrm{PF}}$ for the muons and electrons in the final states $\tau_{\mu} \tau_{\mathrm{h}}$ and $\tau_{\mathrm{e}} \tau_{\mathrm{h}}$ is changed to 0.15 and 0.1 , respectively. In a study of inclusive $\mathrm{Z} \rightarrow \tau \tau$ production [45], it was demonstrated that modifying the muon and electron isolation requirements is a more effective way to reduce background in such final states than requiring tighter isolation on $\tau_{\mathrm{h}}$. The $\tau_{\mathrm{h}}$ are required to have $p_{\mathrm{T}}>20 \mathrm{GeV}$ and $|\eta|<2.3$, and to pass a loose HPS working-point requirement. If the $\mathrm{Z}_{2}$ decays to $\tau_{\mathrm{h}}^{+} \tau_{\mathrm{h}}^{-}$, both $\tau_{\mathrm{h}}$ are required to pass a medium working point of the HPS algorithm. The loose (medium) working point requires the scalar sum over the charged hadrons $p_{\mathrm{T}}$ and 
the neutral hadrons $E_{\mathrm{T}}$ in the isolation cone, to be less than $2 \mathrm{GeV}(1 \mathrm{GeV})$. The loose (medium) working point corresponds to a probability of approximately 1\% (0.5\%) for jets to be misidentified as $\tau_{\mathrm{h}}$. Using the medium instead of loose working point leads to a decrease in the $\tau_{\mathrm{h}}$ reconstruction efficiency from $\approx 50 \%$ to $\approx 40 \%$.

The visible invariant mass of the reconstructed $\mathrm{Z}_{2} \rightarrow \tau^{+} \tau^{-}$is required to be $30<$ $m_{\tau \tau}<80 \mathrm{GeV}$, and this criterion is used for most of the final states. The upper bound reduces contributions from $\mathrm{Z}_{2} \rightarrow \ell \ell$, where a muon or an electron is not well reconstructed, and misidentified as a $\tau_{\mathrm{h}}$. For the $\mathrm{Z}_{2} \rightarrow \tau_{\mathrm{e}} \tau_{\mu}$ final state, the upper bound is increased to $90 \mathrm{GeV}$, as this state is not produced in $\mathrm{Z}_{2} \rightarrow \ell \ell$ decays. Leptons from the same $\mathrm{Z}$ are required to be separated by $\Delta R>0.4$ for $\mathrm{Z}_{1}$, and by $\Delta R>0.5$ for $\mathrm{Z}_{2}$. The two reconstructed $Z_{1}$ and $Z_{2}$ are required to be separated by $\Delta R>0.5$. For a given $m_{\mathrm{H}}, \mathrm{MC}$ simulation predicts that the total number of reconstructed signal events in all $\ell^{+} \ell^{-} \tau^{+} \tau^{-}$ final states is approximately six times smaller than the total number of events in all the $4 \ell$ final states. This difference arises mainly from the higher $p_{\mathrm{T}}$ cuts on the leptons and tighter isolation requirements in $\ell^{+} \ell^{-} \tau^{+} \tau^{-}$, and the $\tau_{\mathrm{h}}$ reconstruction efficiency, which is about one half that of an electron or muon.

A set of Monte Carlo (MC) event samples is used to simulate signal and background events. The Drell-Yan background, $\ell^{+} \ell^{-}$in association with jets, is simulated with the next-to-leading order (NLO) MC generator POWHEG 2.0 [46-48]. The QCD multijet, W and diboson WZ backgrounds are simulated with PYтніA 6.424 [49]. The ZZ background is simulated with PYTHIA 6.424 and MADGRAPH [50]. The t' samples are simulated with MadGraph. The $\tau$-lepton decays are generated with tauola [51]. The Higgs boson samples are generated using POWHEG 2.0, which incorporates NLO gluon fusion $(\mathrm{gg} \rightarrow \mathrm{H})$ and vector-boson fusion ( $\mathrm{qq} \rightarrow \mathrm{qqH}$ ). All events are processed through a detailed simulation of the CMS detector based on GEANT4 [52] and reconstructed with the same algorithms that are used for data.

\section{Background estimates and systematic uncertainties}

The major irreducible source of background to the $\mathrm{H} \rightarrow \mathrm{ZZ} \rightarrow \ell^{+} \ell^{-} \tau^{+} \tau^{-}$process is from $\mathrm{SM} \mathrm{ZZ} \rightarrow \ell^{+} \ell^{-} \tau^{+} \tau^{-}$production. The $\mathrm{ZZ}$ contribution is estimated from data by scaling the prediction from simulation by the ratio of the measured inclusive $\mathrm{Z}$ production cross section to the predicted one. The number of estimated ZZ events, $N_{\mathrm{ZZ}}^{\text {est }}$, can be written as:

$$
N_{\mathrm{ZZ}}^{\mathrm{est}}=N_{\mathrm{Z}}^{\mathrm{obs}} \cdot \frac{\sigma_{\mathrm{ZZ}}^{\mathrm{SM}}}{\sigma_{\mathrm{Z}}^{\mathrm{SM}}} \cdot \frac{A_{\mathrm{ZZ}}}{A_{\mathrm{Z}}}
$$

where $N_{\mathrm{Z}}^{\mathrm{obs}}$ is the number of observed events from inclusive $\mathrm{Z}$ production, $A_{\mathrm{Z}}$ is their estimated acceptance from a $\mathrm{MC}$ simulation, including all selection requirements, and rescaled by measured data/MC correction factors, $A_{\mathrm{ZZ}}$ is the acceptance for $\mathrm{ZZ}$ events, $\sigma_{\mathrm{Z}}^{\mathrm{SM}}$ is the $\mathrm{SM}$ cross section for inclusive $\mathrm{Z}$ production, and $\sigma_{\mathrm{ZZ}}^{\mathrm{SM}}$ is the $\mathrm{SM}$ cross section for ZZ production calculated with MCFM [53].

The other major background contributions arise from the $\mathrm{Z}$ production in association with jets, WZ production in association with jets, $t \bar{t}$, and QCD multijet production. In 
all these cases, a jet or non-isolated lepton is misidentified as a $\tau_{\mathrm{h}}, \tau_{\mathrm{e}}$ or $\tau_{\mu}$. The relative contribution of each source of background depends on the $\ell^{+} \ell^{-} \tau^{+} \tau^{-}$final state.

The probability for jets to be misidentified as $\tau_{\mathrm{h}}$ is measured using $\ell^{+} \ell^{-} \tau_{\mathrm{h}} \tau_{\mathrm{h}}$ events in data in which the $\mathrm{Z}_{1}$ passes all selection requirements, but no requirement is applied on $\tau_{\mathrm{h}}$ isolation, and the two $\tau_{\mathrm{h}}$ candidates are required to have the same charge. This region is dominated by $\mathrm{Z}+$ jets events. The $\tau_{\mathrm{h}}$ misidentification rate is defined as the ratio of the number of $\tau_{\mathrm{h}}$ candidates that pass the HPS loose or medium working-point requirements, to the initial number of $\tau_{\mathrm{h}}$ candidates, and is measured as a function of the $p_{\mathrm{T}}$ for each $\tau_{\mathrm{h}}$. To estimate the number of background events in the signal region, the measured misidentification rate is applied to events that pass all selection requirements, including the opposite-charge requirement for the $\mathrm{Z}_{2}$, but requiring the $\tau_{\mathrm{h}}$ candidates to not be isolated.

The misidentification rate for $\tau_{\mathrm{e}}$ and $\tau_{\mu}$ in the $\mu \mu \tau_{\mu} \tau_{\mathrm{e}}$ and $e e \tau_{\mu} \tau_{\mathrm{e}}$ final states is estimated using events in which the $\mathrm{Z}_{1}$ passes all selection requirements, and the event contains an additional muon or electron. No isolation requirements are applied to it. The misidentification rate is defined for $\tau_{\mathrm{e}}$ and $\tau_{\mu}$ in the same way as described above for $\tau_{\mathrm{h}}$ and applied to $\mu \mu \tau_{\mu} \tau_{\mathrm{e}}$ and $e e \tau_{\mu} \tau_{\mathrm{e}}$ events that pass all the selection requirements, but requiring $\tau_{\mathrm{e}}$ or $\tau_{\mu}$ to not be isolated. Isolated muons and electrons from $\mathrm{H} \rightarrow \mathrm{ZZ} \rightarrow 4 \ell$ and $\mathrm{ZZ} \rightarrow 4 \ell$ production can also be misidentified as $\tau_{\mathrm{h}}$. Events are rejected if they are also identified as $\mathrm{ZZ} \rightarrow 4 \ell$ events with criteria described in ref. [16].

Theoretical uncertainties on the Higgs boson cross section (17-20\%) and branching ratio $(2 \%)$ are taken from ref. [17]. Recent studies [17, 54, 55] show that current MC simulations do not describe the correct Higgs boson mass line shape above $\approx 300 \mathrm{GeV}$. This effect amounts to an additional uncertainty on the theoretical cross section, and hence on the limits, of about $4 \%$ at $m_{\mathrm{H}}=300 \mathrm{GeV}$ and $10-30 \%$ for $m_{\mathrm{H}}$ of $400-600 \mathrm{GeV}$. The main uncertainty on the estimate of the ZZ background arises from the theoretical uncertainty on the ZZ production cross section and is taken from ref. [56]. The uncertainties on the other backgrounds, $\mathrm{Z}+$ jets, $\mathrm{WZ}+$ jets, and $\mathrm{t} \overline{\mathrm{t}}$ reflect the uncertainties on the measured values of the misidentication rates and the limited statistics of the control regions in the data. The uncertainty on integrated luminosity of the data sample is $4.5 \%$ [57]. Systematic uncertainties on trigger efficiency (1\%) and on lepton identification efficiency and isolation are evaluated from data. The uncertainties associated with lepton identification and isolation are $1-2 \%$ for muons and electrons, and $6-7 \%$ for $\tau_{\mathrm{h}}$. Uncertainties on energy scales, $3 \%$ for $\tau_{\mathrm{h}}$ and $1-2.5 \%$ for electrons, contribute to variation in the shape of the mass spectrum.

\section{Results}

Ten $\ell^{+} \ell^{-} \tau^{+} \tau^{-}$candidates are observed in eight search channels, while $11.60 \pm 0.54$ (stat.) \pm 1.62 (syst.) background events are expected. Table 1 compares the estimated number of background events to the number of events observed in the signal region. The distribution of the reconstructed invariant mass summed over all eight $\ell^{+} \ell^{-} \tau^{+} \tau^{-}$decay channels is shown in figure 1. The shape of the background is taken from the MC simulation, with each component normalized to the corresponding estimated value from table 1 . The expected 


\begin{tabular}{|c|c|c|c|c|c|}
\hline $\begin{array}{c}\text { Decay } \\
\text { channel }\end{array}$ & $N_{\mathrm{ZZ}}^{\text {est }}$ & $\begin{array}{c}\text { Other } \\
\text { backgrounds }\end{array}$ & $\begin{array}{c}\text { Total } \\
\text { backgr. }\end{array}$ & $\begin{array}{c}m_{\mathrm{H}} \\
200 \mathrm{GeV}\end{array}$ & Observed \\
\hline$\mu \mu \tau_{\mathrm{h}} \tau_{\mathrm{h}}$ & $0.79 \pm 0.09$ & $0.76 \pm 0.31$ & $1.55 \pm 0.32$ & 0.17 & 0 \\
ee $\tau_{\mathrm{h}} \tau_{\mathrm{h}}$ & $0.75 \pm 0.09$ & $0.73 \pm 0.32$ & $1.48 \pm 0.33$ & 0.15 & 1 \\
ee $\tau_{\mathrm{e}} \tau_{\mathrm{h}}$ & $1.12 \pm 0.13$ & $0.99 \pm 0.34$ & $2.11 \pm 0.36$ & 0.25 & 3 \\
$\mu \mu \tau_{\mathrm{e}} \tau_{\mathrm{h}}$ & $1.20 \pm 0.14$ & $0.31 \pm 0.29$ & $1.51 \pm 0.32$ & 0.26 & 3 \\
$\mu \mu \tau_{\mu} \tau_{\mathrm{h}}$ & $1.08 \pm 0.13$ & $0.67 \pm 0.36$ & $1.75 \pm 0.38$ & 0.23 & 2 \\
ee $\tau_{\mu} \tau_{\mathrm{h}}$ & $0.94 \pm 0.10$ & $0.41 \pm 0.16$ & $1.35 \pm 0.19$ & 0.20 & 0 \\
ee $\tau_{\mathrm{e}} \tau_{\mu}$ & $0.51 \pm 0.06$ & $0.58 \pm 0.42$ & $1.09 \pm 0.42$ & 0.11 & 0 \\
$\mu \mu \tau_{\mathrm{e}} \tau_{\mu}$ & $0.58 \pm 0.07$ & $0.18 \pm 0.18$ & $0.76 \pm 0.22$ & 0.12 & 1 \\
\hline Total & $6.97 \pm 0.84$ & $4.63 \pm 1.49$ & $11.60 \pm 1.71$ & 1.49 & 10 \\
\hline
\end{tabular}

Table 1. The estimated yields of $\mathrm{ZZ}$ and other background events obtained from data, as described in the text, are shown for each decay channel and are summed in the total background yield ("Total backgr."), and compared to the number of events observed in the signal region. The total uncertainty is the sum in quadrature of statistical and systematic uncertainties. The number of signal events expected for the SM Higgs boson with a mass of $m_{\mathrm{H}}=200 \mathrm{GeV}$ is also shown.

mass distributions for the SM Higgs boson with a mass of $m_{\mathrm{H}}=200 \mathrm{GeV}$ and $400 \mathrm{GeV}$ are also shown in figure 1 . The reconstructed masses are shifted with respect to the generated values by $\approx 30 \%$ due to the undetected neutrinos in $\tau$ decays. As a result, the $\mathrm{H} \rightarrow \mathrm{ZZ} \rightarrow \ell^{+} \ell^{-} \tau^{+} \tau^{-}$mass resolution is $10-15 \%$, depending on the final state, and is almost independent of $m_{\mathrm{H}}$.

The product of the acceptance and branching fraction for the individual $\tau$-decay channels ranges between $0.01-0.02$ for $m_{\mathrm{H}}=200 \mathrm{GeV}$, and increases by a factor of three to four for $m_{\mathrm{H}}=400-450 \mathrm{GeV}$. This behaviour is expected. The final-state leptons produced in $\tau$ decays of more massive Higgs bosons have higher momenta than those from direct $\mathrm{Z} \rightarrow \ell \ell$ production and the selection requirements and the lepton reconstruction become more efficient at larger $m_{\mathrm{H}}$. While the cross section decreases with increasing $m_{\mathrm{H}}$, the expected number of Higgs boson events selected by this analysis in $4.7 \mathrm{fb}^{-1}$ of pp collisions at $7 \mathrm{TeV}$ is $1.4-1.5$ in the range $200<m_{\mathrm{H}}<400 \mathrm{GeV}$, and decreases rapidly at higher masses.

In figure 2 the expected and observed upper limits at 95\% CL on the product of the Higgs boson production cross section and decay branching fraction normalized to the SM expectation are presented as a function of $m_{\mathrm{H}}$. The limits are calculated with the modified frequentist construction $\mathrm{CL}_{\mathrm{s}}$ [58-60] based on the shape of the $\ell^{+} \ell^{-} \tau^{+} \tau^{-}$invariant mass distributions by including all eight individual channels in the likelihood combination. The green and yellow bands represent the one- and two-standard-deviation variations from the expected limit. The systematic uncertainties are introduced in the form of nuisance parameters with log-normal probability density functions. The upper limit on the cross section is approximately a factor four to twelve larger than the SM Higgs boson production cross section in the range of $190<m_{\mathrm{H}}<600 \mathrm{GeV}$. 


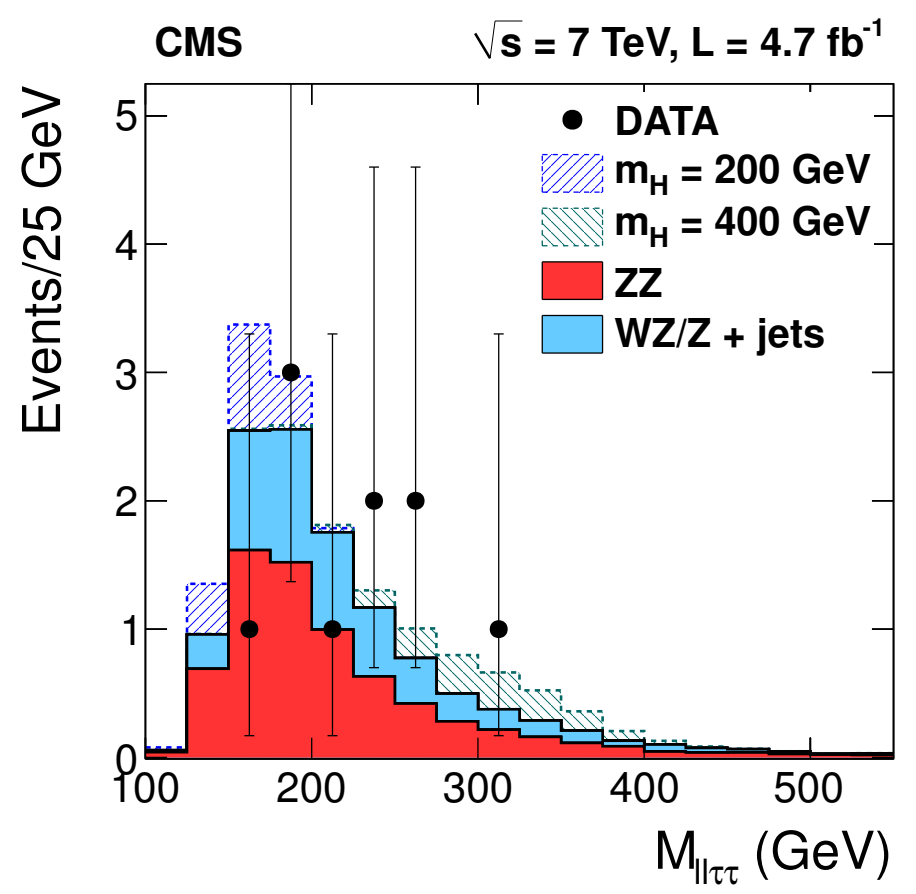

Figure 1. The four-lepton reconstructed mass summed for all $\ell^{+} \ell^{-} \tau^{+} \tau^{-}$final states. The data corresponds to an integrated luminosity of $4.7 \mathrm{fb}^{-1}$. Points represent the data, shaded histograms represent the background and hashed histograms represent the signal expectations for two Higgs boson masses. The background shapes are taken from MC simulation and are normalized to the values obtained using control data samples, as described in the text.

\section{Summary}

A search for the standard model Higgs boson has been performed in the decay mode $\mathrm{H} \rightarrow \mathrm{ZZ} \rightarrow \ell^{+} \ell^{-} \tau^{+} \tau^{-}$using CMS data corresponding to an integrated luminosity of $4.7 \mathrm{fb}^{-1}$. No evidence is found for a significant deviation from the background expectation. An upper limit four to twelve times larger than the predicted value is set at $95 \%$ confidence level for the product of the standard model Higgs boson production cross section and decay branching fraction in the mass range $190<m_{\mathrm{H}}<600 \mathrm{GeV}$. This is the first Higgs boson search performed in the $\mathrm{H} \rightarrow \mathrm{ZZ} \rightarrow \ell^{+} \ell^{-} \tau^{+} \tau^{-}$channel.

\section{Acknowledgments}

We congratulate our colleagues in the CERN accelerator departments for the excellent performance of the LHC machine. We thank the technical and administrative staff at CERN and other CMS institutes, and acknowledge support from: FMSR (Austria); FNRS and FWO (Belgium); CNPq, CAPES, FAPERJ, and FAPESP (Brazil); MES (Bulgaria); CERN; CAS, MoST, and NSFC (China); COLCIENCIAS (Colombia); MSES (Croatia); RPF (Cyprus); MoER, SF0690030s09 and ERDF (Estonia); Academy of Finland, MEC, and HIP (Finland); CEA and CNRS/IN2P3 (France); BMBF, DFG, and HGF (Germany); 


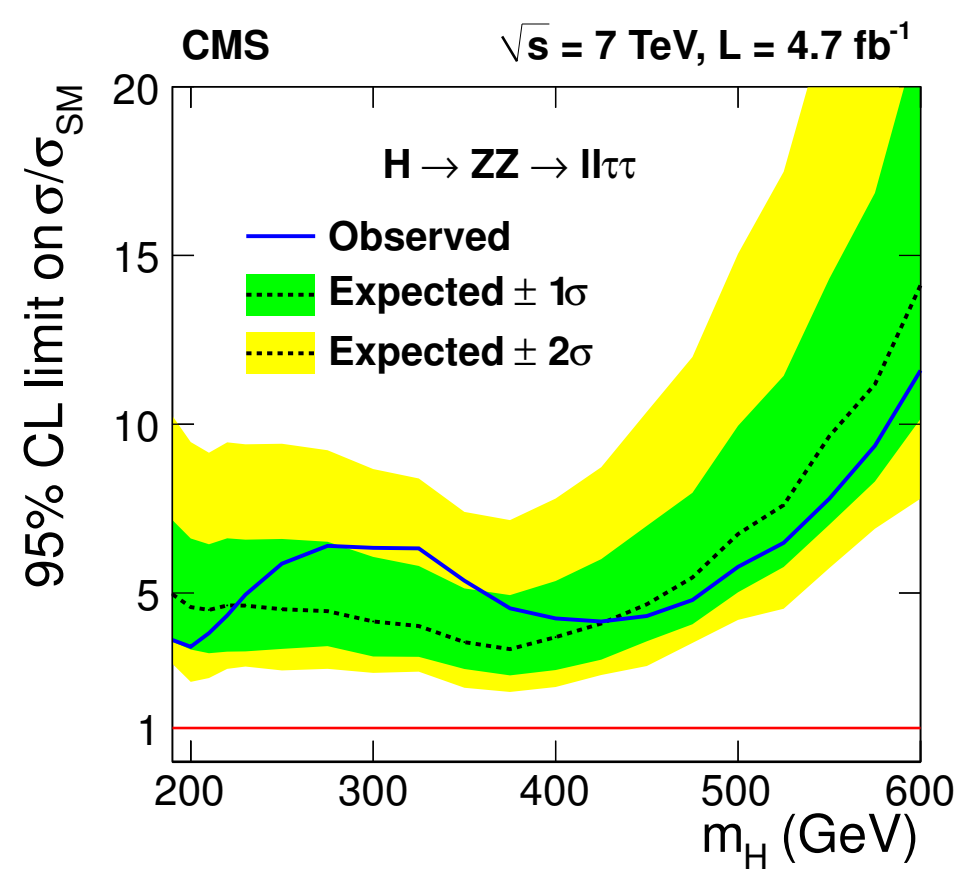

Figure 2. The expected and observed 95\% CL upper limits on the product of the Higgs boson production cross section and decay branching fraction normalized to the SM expectation, denoted by $\sigma / \sigma_{\mathrm{SM}}$, as a function of $m_{\mathrm{H}}$. The one- and two-standard-deviation variations from the expected limit are also shown by the green and yellow bands.

GSRT (Greece); OTKA and NKTH (Hungary); DAE and DST (India); IPM (Iran); SFI (Ireland); INFN (Italy); NRF and WCU (Korea); LAS (Lithuania); CINVESTAV, CONACYT, SEP, and UASLP-FAI (Mexico); MSI (New Zealand); PAEC (Pakistan); MSHE and NSC (Poland); FCT (Portugal); JINR (Armenia, Belarus, Georgia, Ukraine, Uzbekistan); MON, RosAtom, RAS and RFBR (Russia); MSTD (Serbia); MICINN and CPAN (Spain); Swiss Funding Agencies (Switzerland); NSC (Taipei); TUBITAK and TAEK (Turkey); STFC (United Kingdom); DOE and NSF (USA).

Individuals have received support from the Marie-Curie programme and the European Research Council (European Union); the Leventis Foundation; the A. P. Sloan Foundation; the Alexander von Humboldt Foundation; the Belgian Federal Science Policy Office; the Fonds pour la Formation à la Recherche dans l'Industrie et dans l'Agriculture (FRIABelgium); the Agentschap voor Innovatie door Wetenschap en Technologie (IWT-Belgium); the Council of Science and Industrial Research, India; and the HOMING PLUS programme of the Foundation for Polish Science, cofinanced from European Union, Regional Development Fund.

Open Access. This article is distributed under the terms of the Creative Commons Attribution License which permits any use, distribution and reproduction in any medium, provided the original author(s) and source are credited. 


\section{References}

[1] S. Glashow, Partial symmetries of weak interactions, Nucl. Phys. 22 (1961) 579.

[2] S. Weinberg, A model of leptons, Phys. Rev. Lett. 19 (1967) 1264 [InSPIRE].

[3] A. Salam, Weak and electromagnetic interactions, in Elementary particle physics: relativistic groups and analyticity, N. Svartholm ed., Almquvist \& Wiskell International (1968).

[4] F. Englert and R. Brout, Broken symmetry and the mass of gauge vector mesons, Phys. Rev. Lett. 13 (1964) 321 [INSPIRE].

[5] P.W. Higgs, Broken symmetries, massless particles and gauge fields, Phys. Lett. 12 (1964) 132 [INSPIRE].

[6] P.W. Higgs, Broken symmetries and the masses of gauge bosons, Phys. Rev. Lett. 13 (1964) 508 [INSPIRE].

[7] G. Guralnik, C. Hagen and T. Kibble, Global conservation laws and massless particles, Phys. Rev. Lett. 13 (1964) 585 [INSPIRE].

[8] P.W. Higgs, Spontaneous symmetry breakdown without massless bosons, Phys. Rev. 145 (1966) 1156 [INSPIRE].

[9] T. Kibble, Symmetry breaking in non-abelian gauge theories, Phys. Rev. 155 (1967) 1554 [INSPIRE].

[10] LEP Working Group for Higgs boson searches, ALEPH, DELPHI, L3, OPAL collaboration, R. Barate et al., Search for the standard model Higgs boson at LEP, Phys. Lett. B 565 (2003) 61 [hep-ex/0306033] [INSPIRE].

[11] CDF AND D0 collaboration, T. Aaltonen et al., Combined CDF and DO upper limits on standard model Higgs boson production with up to $8.2 \mathrm{fb}^{-1}$ of data, arXiv:1103.3233 [INSPIRE].

[12] ATLAS collaboration, G. Aad et al., Search for the Higgs boson in the $H \rightarrow W W^{*} \rightarrow l \nu l \nu$ decay channel in pp collisions at $\sqrt{s}=7$ TeV with the ATLAS detector, arXiv:1112.2577 [INSPIRE].

[13] ATLAS collaboration, G. Aad et al., Search for the standard model Higgs boson in the decay channel $H \rightarrow Z Z^{*} \rightarrow 4 l$ with the ATLAS detector, Phys. Lett. B 705 (2011) 435 [arXiv: 1109.5945] [INSPIRE].

[14] ATLAS collaboration, Search for a standard model Higgs boson in the $H \rightarrow Z Z \rightarrow l^{+} l^{-} \nu \overline{n u}$ decay channel with the ATLAS detector, Phys. Rev. Lett. 107 (2011) 221802 [arXiv:1109.3357] [INSPIRE].

[15] ALEPH, CDF, D0, DELPHI, L3, OPAL, SLD collaborations, LEP ELECTROWEAK Working Group, Tevatron Electroweak Working Group, and SLD Electroweak and Heavy Flavour Groups, Precision electroweak measurements and constraints on the standard model, PH-EP-2010-095 (2010).

[16] CMS collaboration, S. Chatrchyan et al., Search for the standard model Higgs boson in the decay channel $H$ to $Z Z$ to 4 leptons in pp collisions at $\sqrt{s}=7$ TeV, arXiv:1202.1997 [INSPIRE].

[17] LHC Higgs Cross section Working Group, Handbook of LHC Higgs cross sections: 1. Inclusive observables, arXiv:1101.0593 [CERN-2011-002] [INSPIRE] . 
[18] A. Djouadi, M. Spira and P. Zerwas, Production of Higgs bosons in proton colliders: $Q C D$ corrections, Phys. Lett. B 264 (1991) 440 [INSPIRE].

[19] S. Dawson, Radiative corrections to Higgs boson production, Nucl. Phys. B 359 (1991) 283 [INSPIRE].

[20] M. Spira, A. Djouadi, D. Graudenz and P. Zerwas, Higgs boson production at the LHC, Nucl. Phys. B 453 (1995) 17 [hep-ph/9504378] [INSPIRE].

[21] A. Djouadi, J. Kalinowski and M. Spira, HDECAY: A Program for Higgs boson decays in the standard model and its supersymmetric extension, Comput. Phys. Commun. 108 (1998) 56 [hep-ph/9704448] [INSPIRE].

[22] R.V. Harlander and W.B. Kilgore, Next-to-next-to-leading order Higgs production at hadron colliders, Phys. Rev. Lett. 88 (2002) 201801 [hep-ph/0201206] [INSPIRE].

[23] C. Anastasiou and K. Melnikov, Higgs boson production at hadron colliders in NNLO QCD, Nucl. Phys. B 646 (2002) 220 [hep-ph/0207004] [InSPIRE].

[24] V. Ravindran, J. Smith and W.L. van Neerven, NNLO corrections to the total cross-section for Higgs boson production in hadron hadron collisions, Nucl. Phys. B 665 (2003) 325 [hep-ph/0302135] [INSPIRE].

[25] S. Catani, D. de Florian, M. Grazzini and P. Nason, Soft gluon resummation for Higgs boson production at hadron colliders, JHEP 07 (2003) 028 [hep-ph/0306211] [INSPIRE].

[26] T. Figy, C. Oleari and D. Zeppenfeld, Next-to-leading order jet distributions for Higgs boson production via weak boson fusion, Phys. Rev. D 68 (2003) 073005 [hep-ph/0306109] [INSPIRE].

[27] U. Aglietti, R. Bonciani, G. Degrassi and A. Vicini, Two loop light fermion contribution to Higgs production and decays, Phys. Lett. B 595 (2004) 432 [hep-ph/0404071] [InSPIRE].

[28] G. Degrassi and F. Maltoni, Two-loop electroweak corrections to Higgs production at hadron colliders, Phys. Lett. B 600 (2004) 255 [hep-ph/0407249] [INSPIRE].

[29] A. Bredenstein, A. Denner, S. Dittmaier and M. Weber, Precise predictions for the Higgs-boson decay $H \rightarrow W W / Z Z \rightarrow 4$ leptons, Phys. Rev. D 74 (2006) 013004 [hep-ph/0604011] [INSPIRE].

[30] A. Bredenstein, A. Denner, S. Dittmaier and M. Weber, Radiative corrections to the semileptonic and hadronic Higgs-boson decays $H \rightarrow W W / Z Z \rightarrow 4$ fermions, JHEP 02 (2007) 080 [hep-ph/0611234] [INSPIRE].

[31] M. Ciccolini, A. Denner and S. Dittmaier, Strong and electroweak corrections to the production of Higgs +2 jets via weak interactions at the LHC, Phys. Rev. Lett. 99 (2007) 161803 [arXiv: 0707.0381 ] [INSPIRE].

[32] M. Ciccolini, A. Denner and S. Dittmaier, Electroweak and QCD corrections to Higgs production via vector-boson fusion at the LHC, Phys. Rev. D 77 (2008) 013002 [arXiv: 0710.4749] [INSPIRE].

[33] S. Actis, G. Passarino, C. Sturm and S. Uccirati, NLO electroweak corrections to Higgs boson production at hadron colliders, Phys. Lett. B 670 (2008) 12 [arXiv:0809.1301] [InSPIRE].

[34] C. Anastasiou, R. Boughezal and F. Petriello, Mixed QCD-electroweak corrections to Higgs boson production in gluon fusion, JHEP 04 (2009) 003 [arXiv: 0811.3458] [INSPIRE]. 
[35] D. de Florian and M. Grazzini, Higgs production through gluon fusion: updated cross sections at the Tevatron and the LHC, Phys. Lett. B 674 (2009) 291 [arXiv:0901.2427] [InSPIRE].

[36] P. Bolzoni, F. Maltoni, S.-O. Moch and M. Zaro, Higgs production via vector-boson fusion at NNLO in QCD, Phys. Rev. Lett. 105 (2010) 011801 [arXiv:1003.4451] [INSPIRE].

[37] J. Baglio and A. Djouadi, Higgs production at the LHC, JHEP 03 (2011) 055 [arXiv: 1012.0530] [INSPIRE].

[38] CMS collaboration, S. Chatrchyan et al., The CMS experiment at the CERN LHC, 2008 JINST 3 S08004 [INSPIRE].

[39] CMS collaboration, V. Khachatryan et al., CMS tracking performance results from early LHC operation, Eur. Phys. J. C 70 (2010) 1165 [arXiv: 1007.1988] [InSPIRE].

[40] CMS collaboration, Performance of muon identification in pp collisions at $\sqrt{s}=7 \mathrm{TeV}$, PAS-MUO-10-002 (2010).

[41] CMS collaboration, Electron reconstruction and identification at $\sqrt{(} s)=7 \mathrm{TeV}$, PAS-EGM-10-004 (2010).

[42] CMS collaboration, Particle-flow event reconstruction in CMS and performance for jets, taus and MET, PAS-PFT-09-001 (2009).

[43] CMS collaboration, Performance of tau-lepton reconstruction and identification in CMS, 2012 JINST 7 P01001 [arXiv:1109.6034] [INSPIRE].

[44] CMS collaboration, G. Bayatian et al., CMS technical design report, volume II: Physics performance, J. Phys. G 34 (2007) 995 [inSPIRE].

[45] CMS collaboration, S. Chatrchyan et al., Measurement of the inclusive $Z$ cross section via decays to tau pairs in pp collisions at $\sqrt{s}=7$ TeV, JHEP 08 (2011) 117 [arXiv:1104.1617] [INSPIRE].

[46] S. Alioli, P. Nason, C. Oleari and E. Re, NLO vector-boson production matched with shower in POWHEG, JHEP 07 (2008) 060 [arXiv:0805.4802] [INSPIRE].

[47] P. Nason, A new method for combining NLO QCD with shower Monte Carlo algorithms, JHEP 11 (2004) 040 [hep-ph/0409146] [INSPIRE].

[48] S. Frixione, P. Nason and C. Oleari, Matching NLO QCD computations with parton shower simulations: the POWHEG method, JHEP 11 (2007) 070 [arXiv: 0709.2092] [INSPIRE].

[49] T. Sjöstrand, S. Mrenna and P.Z. Skands, PYTHIA 6.4 physics and manual, JHEP 05 (2006) 026 [hep-ph/0603175] [INSPIRE].

[50] F. Maltoni and T. Stelzer, MadEvent: automatic event generation with MadGraph, JHEP 02 (2003) 027 [hep-ph/0208156] [inSPIRE].

[51] S. Jadach, Z. Was, R. Decker and J.H. Kuhn, The $\tau$ decay library TAUOLA: version 2.4, Comput. Phys. Commun. 76 (1993) 361 [InSPIRE].

[52] J. Allison et al., Geant4 developments and applications, IEEE Trans. Nucl. Sci. 53 (2006) 270.

[53] J.M. Campbell and R. Ellis, MCFM for the Tevatron and the LHC, Nucl. Phys. Proc. Suppl. 205-206 (2010) 10 [arXiv: 1007.3492] [InSPIRE].

[54] G. Passarino, C. Sturm and S. Uccirati, Higgs pseudo-observables, second Riemann sheet and all that, Nucl. Phys. B 834 (2010) 77 [arXiv:1001.3360] [InSPIRE]. 
[55] C. Anastasiou, S. Buehler, F. Herzog and A. Lazopoulos, Total cross-section for Higgs boson hadroproduction with anomalous Standard Model interactions, JHEP 12 (2011) 058 [arXiv: 1107.0683] [INSPIRE].

[56] LHC Higgs Cross section Working Group collaboration, Handbook of LHC Higgs cross sections: 2 Differential distributions, CERN-2012-002 (2012).

[57] CMS collaboration, Absolute calibration of luminosity measurement at CMS: summer 2011 update, PAS-EWK-11-001 (2011).

[58] T. Junk, Confidence level computation for combining searches with small statistics, Nucl. Instrum. Meth. A 434 (1999) 435 [hep-ex/9902006] [INSPIRE].

[59] A.L. Read, A search for heavy stable charged and long-lived squarks and sleptons in $e^{+} e^{-}$ collisions at energies from 130 to 183 GeV, CERN-OPEN-2000-005 (2000).

[60] ATLAS and CMS collaboration, Procedure for the LHC Higgs boson search combination in summer 2011, PHYS-PUB-2011-011 (2011). 


\section{The CMS collaboration}

\section{Yerevan Physics Institute, Yerevan, Armenia}

S. Chatrchyan, V. Khachatryan, A.M. Sirunyan, A. Tumasyan

\section{Institut für Hochenergiephysik der OeAW, Wien, Austria}

W. Adam, T. Bergauer, M. Dragicevic, J. Erö, C. Fabjan, M. Friedl, R. Frühwirth, V.M. Ghete, J. Hammer ${ }^{1}$, M. Hoch, N. Hörmann, J. Hrubec, M. Jeitler, W. Kiesenhofer, M. Krammer, D. Liko, I. Mikulec, M. Pernicka ${ }^{\dagger}$, B. Rahbaran, C. Rohringer, H. Rohringer, R. Schöfbeck, J. Strauss, A. Taurok, F. Teischinger, P. Wagner, W. Waltenberger, G. Walzel, E. Widl, C.-E. Wulz

National Centre for Particle and High Energy Physics, Minsk, Belarus

V. Mossolov, N. Shumeiko, J. Suarez Gonzalez

\section{Universiteit Antwerpen, Antwerpen, Belgium}

S. Bansal, L. Benucci, T. Cornelis, E.A. De Wolf, X. Janssen, S. Luyckx, T. Maes, L. Mucibello, S. Ochesanu, B. Roland, R. Rougny, M. Selvaggi, H. Van Haevermaet, P. Van Mechelen, N. Van Remortel, A. Van Spilbeeck

\section{Vrije Universiteit Brussel, Brussel, Belgium}

F. Blekman, S. Blyweert, J. D'Hondt, R. Gonzalez Suarez, A. Kalogeropoulos, M. Maes, A. Olbrechts, W. Van Doninck, P. Van Mulders, G.P. Van Onsem, I. Villella

\section{Université Libre de Bruxelles, Bruxelles, Belgium}

O. Charaf, B. Clerbaux, G. De Lentdecker, V. Dero, A.P.R. Gay, G.H. Hammad, T. Hreus, A. Léonard, P.E. Marage, L. Thomas, C. Vander Velde, P. Vanlaer, J. Wickens

\section{Ghent University, Ghent, Belgium}

V. Adler, K. Beernaert, A. Cimmino, S. Costantini, G. Garcia, M. Grunewald, B. Klein, J. Lellouch, A. Marinov, J. Mccartin, A.A. Ocampo Rios, D. Ryckbosch, N. Strobbe, F. Thyssen, M. Tytgat, L. Vanelderen, P. Verwilligen, S. Walsh, E. Yazgan, N. Zaganidis

Université Catholique de Louvain, Louvain-la-Neuve, Belgium

S. Basegmez, G. Bruno, L. Ceard, J. De Favereau De Jeneret, C. Delaere, T. du Pree, D. Favart, L. Forthomme, A. Giammanco ${ }^{2}$, G. Grégoire, J. Hollar, V. Lemaitre, J. Liao, O. Militaru, C. Nuttens, D. Pagano, A. Pin, K. Piotrzkowski, N. Schul

\section{Université de Mons, Mons, Belgium}

N. Beliy, T. Caebergs, E. Daubie

\section{Centro Brasileiro de Pesquisas Fisicas, Rio de Janeiro, Brazil}

G.A. Alves, M. Correa Martins Junior, D. De Jesus Damiao, T. Martins, M.E. Pol, M.H.G. Souza

\section{Universidade do Estado do Rio de Janeiro, Rio de Janeiro, Brazil}

W.L. Aldá Júnior, W. Carvalho, A. Custódio, E.M. Da Costa, C. De Oliveira Martins, S. Fonseca De Souza, D. Matos Figueiredo, L. Mundim, H. Nogima, V. Oguri, W.L. Prado Da Silva, A. Santoro, S.M. Silva Do Amaral, L. Soares Jorge, A. Sznajder 
Instituto de Fisica Teorica, Universidade Estadual Paulista, Sao Paulo, Brazil T.S. Anjos $^{3}$, C.A. Bernardes ${ }^{3}$, F.A. Dias ${ }^{4}$, T.R. Fernandez Perez Tomei, E. M. Gregores ${ }^{3}$, C. Lagana, F. Marinho, P.G. Mercadante ${ }^{3}$, S.F. Novaes, Sandra S. Padula

Institute for Nuclear Research and Nuclear Energy, Sofia, Bulgaria

V. Genchev ${ }^{1}$, P. Iaydjiev ${ }^{1}$, S. Piperov, M. Rodozov, S. Stoykova, G. Sultanov, V. Tcholakov,

R. Trayanov, M. Vutova

University of Sofia, Sofia, Bulgaria

A. Dimitrov, R. Hadjiiska, A. Karadzhinova, V. Kozhuharov, L. Litov, B. Pavlov, P. Petkov

Institute of High Energy Physics, Beijing, China

J.G. Bian, G.M. Chen, H.S. Chen, C.H. Jiang, D. Liang, S. Liang, X. Meng, J. Tao, J. Wang, J. Wang, X. Wang, Z. Wang, H. Xiao, M. Xu, J. Zang, Z. Zhang

State Key Lab. of Nucl. Phys. and Tech., Peking University, Beijing, China C. Asawatangtrakuldee, Y. Ban, S. Guo, Y. Guo, W. Li, S. Liu, Y. Mao, S.J. Qian, H. Teng, S. Wang, B. Zhu, W. Zou

Universidad de Los Andes, Bogota, Colombia

A. Cabrera, B. Gomez Moreno, A.F. Osorio Oliveros, J.C. Sanabria

Technical University of Split, Split, Croatia

N. Godinovic, D. Lelas, R. Plestina ${ }^{5}$, D. Polic, I. Puljak ${ }^{1}$

University of Split, Split, Croatia

Z. Antunovic, M. Dzelalija, M. Kovac

Institute Rudjer Boskovic, Zagreb, Croatia

V. Brigljevic, S. Duric, K. Kadija, J. Luetic, S. Morovic

University of Cyprus, Nicosia, Cyprus

A. Attikis, M. Galanti, J. Mousa, C. Nicolaou, F. Ptochos, P.A. Razis

Charles University, Prague, Czech Republic

M. Finger, M. Finger Jr.

Academy of Scientific Research and Technology of the Arab Republic of Egypt, Egyptian Network of High Energy Physics, Cairo, Egypt

Y. Assran ${ }^{6}$, A. Ellithi Kamel ${ }^{7}$, S. Khalil ${ }^{8}$, M.A. Mahmoud ${ }^{9}$, A. Radi ${ }^{8,10}$

National Institute of Chemical Physics and Biophysics, Tallinn, Estonia

A. Hektor, M. Kadastik, M. Müntel, M. Raidal, L. Rebane, A. Tiko

Department of Physics, University of Helsinki, Helsinki, Finland

V. Azzolini, P. Eerola, G. Fedi, M. Voutilainen

Helsinki Institute of Physics, Helsinki, Finland

S. Czellar, J. Härkönen, A. Heikkinen, V. Karimäki, R. Kinnunen, M.J. Kortelainen,

T. Lampén, K. Lassila-Perini, S. Lehti, T. Lindén, P. Luukka, T. Mäenpää, T. Peltola,

E. Tuominen, J. Tuominiemi, E. Tuovinen, D. Ungaro, L. Wendland 
Lappeenranta University of Technology, Lappeenranta, Finland

K. Banzuzi, A. Korpela, T. Tuuva

Laboratoire d'Annecy-le-Vieux de Physique des Particules, IN2P3-CNRS, Annecy-le-Vieux, France

D. Sillou

\section{DSM/IRFU, CEA/Saclay, Gif-sur-Yvette, France}

M. Besancon, S. Choudhury, M. Dejardin, D. Denegri, B. Fabbro, J.L. Faure, F. Ferri, S. Ganjour, A. Givernaud, P. Gras, G. Hamel de Monchenault, P. Jarry, E. Locci, J. Malcles, L. Millischer, J. Rander, A. Rosowsky, I. Shreyber, M. Titov

Laboratoire Leprince-Ringuet, Ecole Polytechnique, IN2P3-CNRS, Palaiseau, France

S. Baffioni, F. Beaudette, L. Benhabib, L. Bianchini, M. Bluj ${ }^{11}$, C. Broutin, P. Busson, C. Charlot, N. Daci, T. Dahms, L. Dobrzynski, S. Elgammal, R. Granier de Cassagnac, M. Haguenauer, P. Miné, C. Mironov, C. Ochando, P. Paganini, D. Sabes, R. Salerno, Y. Sirois, C. Thiebaux, C. Veelken, A. Zabi

Institut Pluridisciplinaire Hubert Curien, Université de Strasbourg, Université de Haute Alsace Mulhouse, CNRS/IN2P3, Strasbourg, France

J.-L. Agram ${ }^{12}$, J. Andrea, D. Bloch, D. Bodin, J.-M. Brom, M. Cardaci, E.C. Chabert, C. Collard, E. Conte ${ }^{12}$, F. Drouhin ${ }^{12}$, C. Ferro, J.-C. Fontaine ${ }^{12}$, D. Gelé, U. Goerlach, P. Juillot, M. Karim ${ }^{12}$, A.-C. Le Bihan, P. Van Hove

Centre de Calcul de l'Institut National de Physique Nucleaire et de Physique des Particules (IN2P3), Villeurbanne, France

F. Fassi, D. Mercier

Université de Lyon, Université Claude Bernard Lyon 1, CNRS-IN2P3, Institut de Physique Nucléaire de Lyon, Villeurbanne, France

C. Baty, S. Beauceron, N. Beaupere, M. Bedjidian, O. Bondu, G. Boudoul, D. Boumediene, H. Brun, J. Chasserat, R. Chierici ${ }^{1}$, D. Contardo, P. Depasse, H. El Mamouni, A. Falkiewicz, J. Fay, S. Gascon, M. Gouzevitch, B. Ille, T. Kurca, T. Le Grand, M. Lethuillier, L. Mirabito, S. Perries, V. Sordini, S. Tosi, Y. Tschudi, P. Verdier, S. Viret

Institute of High Energy Physics and Informatization, Tbilisi State University, Tbilisi, Georgia

D. Lomidze

RWTH Aachen University, I. Physikalisches Institut, Aachen, Germany

G. Anagnostou, S. Beranek, M. Edelhoff, L. Feld, N. Heracleous, O. Hindrichs, R. Jussen, K. Klein, J. Merz, A. Ostapchuk, A. Perieanu, F. Raupach, J. Sammet, S. Schael, D. Sprenger, H. Weber, B. Wittmer, V. Zhukov ${ }^{13}$

RWTH Aachen University, III. Physikalisches Institut A, Aachen, Germany M. Ata, J. Caudron, E. Dietz-Laursonn, M. Erdmann, A. Güth, T. Hebbeker, C. Heidemann, K. Hoepfner, T. Klimkovich, D. Klingebiel, P. Kreuzer, D. Lanske ${ }^{\dagger}$, J. Lingemann, 
C. Magass, M. Merschmeyer, A. Meyer, M. Olschewski, P. Papacz, H. Pieta, H. Reithler, S.A. Schmitz, L. Sonnenschein, J. Steggemann, D. Teyssier, M. Weber

\section{RWTH Aachen University, III. Physikalisches Institut B, Aachen, Germany}

M. Bontenackels, V. Cherepanov, M. Davids, G. Flügge, H. Geenen, M. Geisler, W. Haj Ahmad, F. Hoehle, B. Kargoll, T. Kress, Y. Kuessel, A. Linn, A. Nowack, L. Perchalla, O. Pooth, J. Rennefeld, P. Sauerland, A. Stahl, M.H. Zoeller

\section{Deutsches Elektronen-Synchrotron, Hamburg, Germany}

M. Aldaya Martin, W. Behrenhoff, U. Behrens, M. Bergholz ${ }^{14}$, A. Bethani, K. Borras, A. Burgmeier, A. Cakir, L. Calligaris, A. Campbell, E. Castro, D. Dammann, G. Eckerlin, D. Eckstein, A. Flossdorf, G. Flucke, A. Geiser, J. Hauk, H. Jung ${ }^{1}$, M. Kasemann, P. Katsas, C. Kleinwort, H. Kluge, A. Knutsson, M. Krämer, D. Krücker, E. Kuznetsova, W. Lange, W. Lohmann ${ }^{14}$, B. Lutz, R. Mankel, I. Marfin, M. Marienfeld, I.-A. Melzer-Pellmann, A.B. Meyer, J. Mnich, A. Mussgiller, S. Naumann-Emme, J. Olzem, A. Petrukhin, D. Pitzl, A. Raspereza, P.M. Ribeiro Cipriano, M. Rosin, J. Salfeld-Nebgen, R. Schmidt ${ }^{14}$, T. Schoerner-Sadenius, N. Sen, A. Spiridonov, M. Stein, J. Tomaszewska, R. Walsh, C. Wissing

\section{University of Hamburg, Hamburg, Germany}

C. Autermann, V. Blobel, S. Bobrovskyi, J. Draeger, H. Enderle, J. Erfle, U. Gebbert, M. Görner, T. Hermanns, R.S. Höing, K. Kaschube, G. Kaussen, H. Kirschenmann, R. Klanner, J. Lange, B. Mura, F. Nowak, N. Pietsch, C. Sander, H. Schettler, P. Schleper, E. Schlieckau, A. Schmidt, M. Schröder, T. Schum, H. Stadie, G. Steinbrück, J. Thomsen

\section{Institut für Experimentelle Kernphysik, Karlsruhe, Germany}

C. Barth, J. Berger, T. Chwalek, W. De Boer, A. Dierlamm, G. Dirkes, M. Feindt, J. Gruschke, M. Guthoff ${ }^{1}$, C. Hackstein, F. Hartmann, M. Heinrich, H. Held, K.H. Hoffmann, S. Honc, I. Katkov ${ }^{13}$, J.R. Komaragiri, T. Kuhr, D. Martschei, S. Mueller, Th. Müller, M. Niegel, A. Nürnberg, O. Oberst, A. Oehler, J. Ott, T. Peiffer, G. Quast, K. Rabbertz, F. Ratnikov, N. Ratnikova, M. Renz, S. Röcker, C. Saout, A. Scheurer, P. Schieferdecker, F.-P. Schilling, M. Schmanau, G. Schott, H.J. Simonis, F.M. Stober, D. Troendle, J. Wagner-Kuhr, T. Weiler, M. Zeise, E.B. Ziebarth

\section{Institute of Nuclear Physics "Demokritos", Aghia Paraskevi, Greece}

G. Daskalakis, T. Geralis, S. Kesisoglou, A. Kyriakis, D. Loukas, I. Manolakos, A. Markou, C. Markou, C. Mavrommatis, E. Ntomari

\section{University of Athens, Athens, Greece}

L. Gouskos, T.J. Mertzimekis, A. Panagiotou, N. Saoulidou, E. Stiliaris

University of Ioánnina, Ioánnina, Greece

I. Evangelou, C. Foudas ${ }^{1}$, P. Kokkas, N. Manthos, I. Papadopoulos, V. Patras, F.A. Triantis

KFKI Research Institute for Particle and Nuclear Physics, Budapest, Hungary

A. Aranyi, G. Bencze, L. Boldizsar, C. Hajdu ${ }^{1}$, P. Hidas, D. Horvath ${ }^{15}$, A. Kapusi, K. Krajczar ${ }^{16}$, F. Sikler ${ }^{1}$, V. Veszpremi, G. Vesztergombi ${ }^{16}$ 
Institute of Nuclear Research ATOMKI, Debrecen, Hungary

N. Beni, J. Molnar, J. Palinkas, Z. Szillasi

University of Debrecen, Debrecen, Hungary

J. Karancsi, P. Raics, Z.L. Trocsanyi, B. Ujvari

Panjab University, Chandigarh, India

S.B. Beri, V. Bhatnagar, N. Dhingra, R. Gupta, M. Jindal, M. Kaur, J.M. Kohli, M.Z. Mehta, N. Nishu, L.K. Saini, A. Sharma, A.P. Singh, J. Singh, S.P. Singh

University of Delhi, Delhi, India

S. Ahuja, B.C. Choudhary, A. Kumar, A. Kumar, S. Malhotra, M. Naimuddin, K. Ranjan, V. Sharma, R.K. Shivpuri

Saha Institute of Nuclear Physics, Kolkata, India

S. Banerjee, S. Bhattacharya, S. Dutta, B. Gomber, S. Jain, S. Jain, R. Khurana, S. Sarkar

Bhabha Atomic Research Centre, Mumbai, India

R.K. Choudhury, D. Dutta, S. Kailas, V. Kumar, A.K. Mohanty ${ }^{1}$, L.M. Pant, P. Shukla

Tata Institute of Fundamental Research - EHEP, Mumbai, India

T. Aziz, S. Ganguly, M. Guchait ${ }^{17}$, A. Gurtu ${ }^{18}$, M. Maity ${ }^{19}$, G. Majumder, K. Mazumdar,

G.B. Mohanty, B. Parida, A. Saha, K. Sudhakar, N. Wickramage

Tata Institute of Fundamental Research - HECR, Mumbai, India

S. Banerjee, S. Dugad, N.K. Mondal

Institute for Research in Fundamental Sciences (IPM), Tehran, Iran

H. Arfaei, H. Bakhshiansohi ${ }^{20}$, S.M. Etesami ${ }^{21}$, A. Fahim ${ }^{20}$, M. Hashemi, H. Hesari, A. Jafari ${ }^{20}$, M. Khakzad, A. Mohammadi ${ }^{22}$, M. Mohammadi Najafabadi, S. Paktinat Mehdiabadi, B. Safarzadeh ${ }^{23}$, M. Zeinali ${ }^{21}$

INFN Sezione di Bari ${ }^{a}$, Università di Bari ${ }^{b}$, Politecnico di Bari ${ }^{c}$, Bari, Italy M. Abbrescia ${ }^{a, b}$, L. Barbone ${ }^{a, b}$, C. Calabria ${ }^{a, b}$, S.S. Chhibra ${ }^{a, b}$, A. Colaleo $^{a}$, D. Creanza $^{a, c}$, N. De Filippis ${ }^{a, c, 1}$, M. De Palma ${ }^{a, b}$, L. Fiore $^{a}$, G. Iaselli ${ }^{a, c}$, L. Lusito $^{a, b}$, G. Maggi $^{a, c}$, M. Maggi ${ }^{a}$, N. Manna ${ }^{a, b}$, B. Marangellia ${ }^{a, b}$, S. My ${ }^{a, c}$, S. Nuzzo ${ }^{a, b}$, N. Pacifico ${ }^{a, b}$,

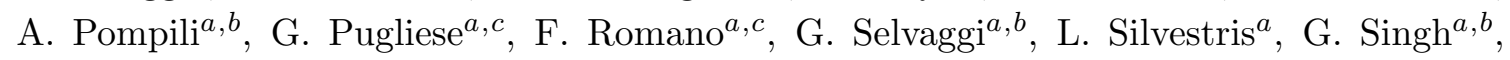
S. Tupputi ${ }^{a, b}$, G. Zito ${ }^{a}$

INFN Sezione di Bologna ${ }^{a}$, Università di Bologna ${ }^{b}$, Bologna, Italy

G. Abbiendi ${ }^{a}$, A.C. Benvenuti ${ }^{a}$, D. Bonacorsi ${ }^{a}$, S. Braibant-Giacomelli ${ }^{a, b}$, L. Brigliadori ${ }^{a}$, P. Capiluppi ${ }^{a, b}$, A. Castro ${ }^{a, b}$, F.R. Cavallo ${ }^{a}$, M. Cuffiani ${ }^{a, b}$, G.M. Dallavalle ${ }^{a}$, F. Fabbri ${ }^{a}$, A. Fanfani ${ }^{a}, b$, D. Fasanella ${ }^{a, 1}$, P. Giacomelli ${ }^{a}$, C. Grandi ${ }^{a}$, S. Marcellini ${ }^{a}$,

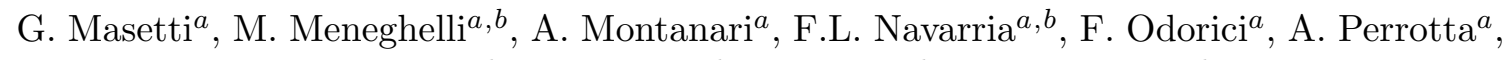

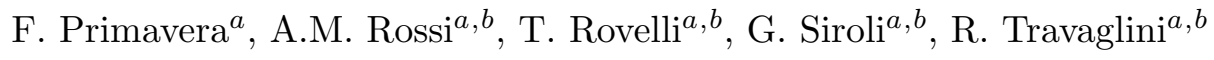

INFN Sezione di Catania ${ }^{a}$, Università di Catania ${ }^{b}$, Catania, Italy

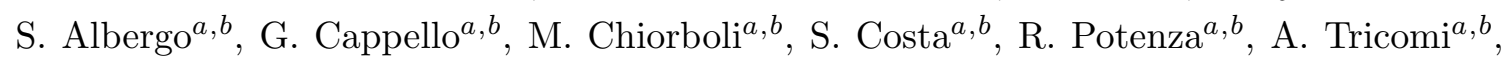
C. Tuve ${ }^{a, b}$ 
INFN Sezione di Firenze ${ }^{a}$, Università di Firenze ${ }^{b}$, Firenze, Italy

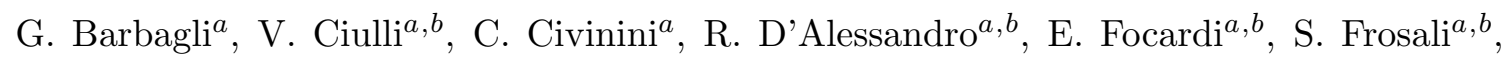

E. Gallo ${ }^{a}$, S. Gonzi ${ }^{a}, b$, M. Meschini ${ }^{a}$, S. Paoletti ${ }^{a}$, G. Sguazzoni ${ }^{a}$, A. Tropiano ${ }^{a, 1}$

INFN Laboratori Nazionali di Frascati, Frascati, Italy

L. Benussi, S. Bianco, S. Colafranceschi ${ }^{24}$, F. Fabbri, D. Piccolo

INFN Sezione di Genova, Genova, Italy

P. Fabbricatore, R. Musenich

INFN Sezione di Milano-Bicocca ${ }^{a}$, Università di Milano-Bicocca ${ }^{b}$, Milano, Italy

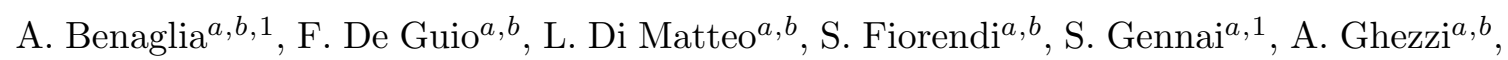
S. Malvezzi ${ }^{a}$, R.A. Manzoni ${ }^{a, b}$, A. Martelli ${ }^{a}, b$, A. Massironi ${ }^{a, b, 1}$, D. Menasce ${ }^{a}$, L. Moroni ${ }^{a}$, M. Paganoni ${ }^{a, b}$, D. Pedrini ${ }^{a}$, S. Ragazzi ${ }^{a, b}$, N. Redaelli ${ }^{a}$, S. Sala ${ }^{a}$, T. Tabarelli de Fatis ${ }^{a, b}$

INFN Sezione di Napoli ${ }^{a}$, Università di Napoli "Federico II" ${ }^{b}$, Napoli, Italy S. Buontempo ${ }^{a}$, C.A. Carrillo Montoya ${ }^{a, 1}$, N. Cavallo ${ }^{a, 25}$, A. De Cosa $^{a, b}$, O. Dogangun ${ }^{a, b}$,

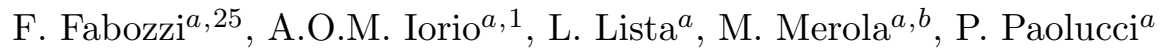

INFN Sezione di Padova ${ }^{a}$, Università di Padova ${ }^{b}$, Università di Trento (Trento) ${ }^{c}$, Padova, Italy

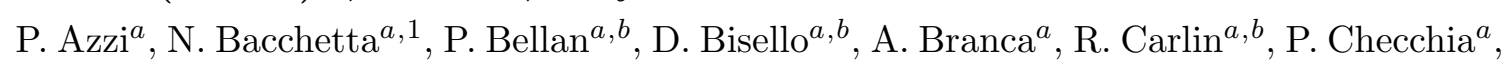

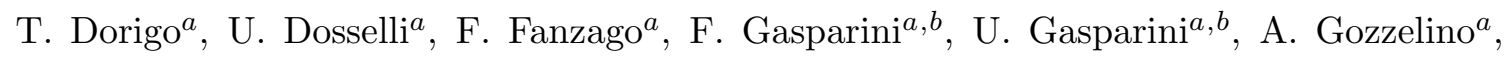
K. Kanishchev, S. Lacaprara ${ }^{a, 26}$, I. Lazzizzera ${ }^{a, c}$, M. Margoni ${ }^{a, b}$, M. Mazzucato ${ }^{a}$, A.T. Meneguzzo ${ }^{a, b}$, M. Nespolo ${ }^{a, 1}$, L. Perrozzi ${ }^{a}$, N. Pozzobon ${ }^{a, b}$, P. Ronchese ${ }^{a, b}$,

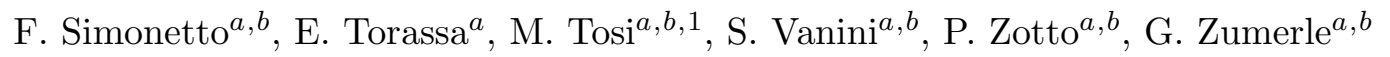

INFN Sezione di Pavia ${ }^{a}$, Università di Pavia ${ }^{b}$, Pavia, Italy

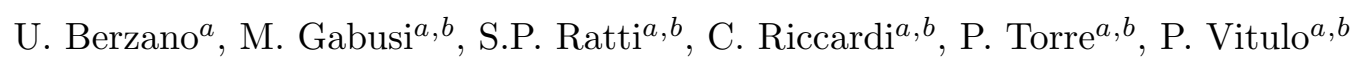

INFN Sezione di Perugia ${ }^{a}$, Università di Perugia ${ }^{b}$, Perugia, Italy

M. Biasini ${ }^{a, b}$, G.M. Bilei ${ }^{a}$, B. Caponeri ${ }^{a, b}$, L. Fanò ${ }^{a, b}$, P. Lariccia ${ }^{a, b}$, A. Lucaroni ${ }^{a, b, 1}$, G. Mantovani ${ }^{a, b}$, M. Menichelli ${ }^{a}$, A. Nappi $^{a, b}$, F. Romeo $^{a, b}$, A. Santocchia $^{a, b}$, S. Taroni ${ }^{a, b, 1}$, M. Valdata ${ }^{a, b}$

INFN Sezione di Pisa ${ }^{a}$, Università di Pisa ${ }^{b}$, Scuola Normale Superiore di Pisa ${ }^{c}$, Pisa, Italy

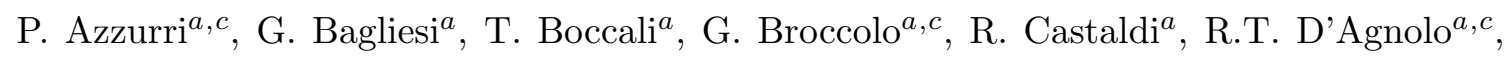

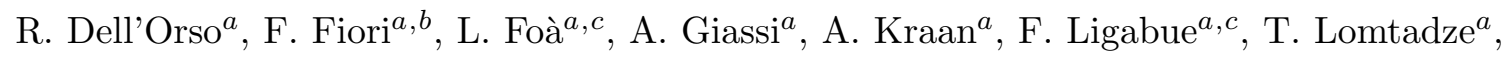
L. Martini ${ }^{a, 27}$, A. Messineo ${ }^{a, b}$, F. Palla ${ }^{a}$, F. Palmonari ${ }^{a}$, A. Rizzi, A.T. Serban ${ }^{a}$,

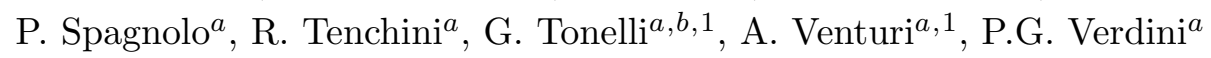

INFN Sezione di Roma ${ }^{a}$, Università di Roma "La Sapienza" ${ }^{b}$, Roma, Italy

L. Barone $e^{a, b}$, F. Cavallari ${ }^{a}$, D. Del Re $e^{a, b, 1}$, M. Diemoz ${ }^{a}$, C. Fanelli, M. Grassi ${ }^{a, 1}$, E. Longo ${ }^{a, b}$, P. Meridiani ${ }^{a}$, F. Micheli, S. Nourbakhsh ${ }^{a}$, G. Organtini ${ }^{a, b}$, F. Pandolfi ${ }^{a, b}$, R. Paramatti ${ }^{a}$, S. Rahatlou ${ }^{a, b}$, M. Sigamani ${ }^{a}$, L. Soffi 
INFN Sezione di Torino ${ }^{a}$, Università di Torino ${ }^{b}$, Università del Piemonte Orientale (Novara) ${ }^{c}$, Torino, Italy

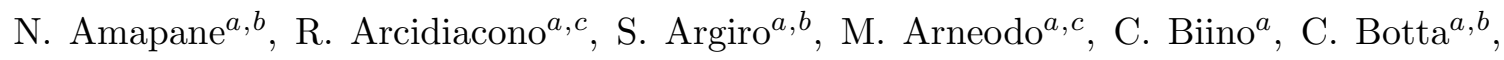

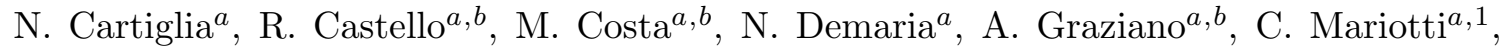
S. Maselli ${ }^{a}$, E. Migliore ${ }^{a, b}$, V. Monaco ${ }^{a, b}$, M. Musich $^{a}$, M.M. Obertino $^{a, c}$, N. Pastrone ${ }^{a}$, M. Pelliccioni ${ }^{a}$, A. Potenza ${ }^{a, b}$, A. Romero ${ }^{a, b}$, M. Ruspa ${ }^{a, c}$, R. Sacchi ${ }^{a, b}$, V. Sola $^{a, b}$, A. Solano $^{a, b}$, A. Staiano ${ }^{a}$, A. Vilela Pereira ${ }^{a}$

INFN Sezione di Trieste ${ }^{a}$, Università di Trieste ${ }^{b}$, Trieste, Italy

S. Belforte ${ }^{a}$, F. Cossutti $^{a}$, G. Della Ricca ${ }^{a, b}$, B. Gobbo ${ }^{a}$, M. Marone $^{a, b}$, D. Montanino $^{a, b, 1}$, A. Penzo ${ }^{a}$

Kangwon National University, Chunchon, Korea

S.G. Heo, S.K. Nam

Kyungpook National University, Daegu, Korea

S. Chang, J. Chung, D.H. Kim, G.N. Kim, J.E. Kim, D.J. Kong, H. Park, S.R. Ro, D.C. Son

Chonnam National University, Institute for Universe and Elementary Particles, Kwangju, Korea

J.Y. Kim, Zero J. Kim, S. Song

Konkuk University, Seoul, Korea

H.Y. Jo

Korea University, Seoul, Korea

S. Choi, D. Gyun, B. Hong, M. Jo, H. Kim, T.J. Kim, K.S. Lee, D.H. Moon, S.K. Park, E. Seo, K.S. Sim

University of Seoul, Seoul, Korea

M. Choi, S. Kang, H. Kim, J.H. Kim, C. Park, I.C. Park, S. Park, G. Ryu

Sungkyunkwan University, Suwon, Korea

Y. Cho, Y. Choi, Y.K. Choi, J. Goh, M.S. Kim, B. Lee, J. Lee, S. Lee, H. Seo, I. Yu

Vilnius University, Vilnius, Lithuania

M.J. Bilinskas, I. Grigelionis, M. Janulis

Centro de Investigacion y de Estudios Avanzados del IPN, Mexico City, Mexico

H. Castilla-Valdez, E. De La Cruz-Burelo, I. Heredia-de La Cruz, R. Lopez-Fernandez,

R. Magaña Villalba, J. Martínez-Ortega, A. Sánchez-Hernández, L.M. Villasenor-Cendejas

Universidad Iberoamericana, Mexico City, Mexico

S. Carrillo Moreno, F. Vazquez Valencia

Benemerita Universidad Autonoma de Puebla, Puebla, Mexico

H.A. Salazar Ibarguen

Universidad Autónoma de San Luis Potosí, San Luis Potosí, Mexico

E. Casimiro Linares, A. Morelos Pineda, M.A. Reyes-Santos 
University of Auckland, Auckland, New Zealand

D. Krofcheck

University of Canterbury, Christchurch, New Zealand

A.J. Bell, P.H. Butler, R. Doesburg, S. Reucroft, H. Silverwood

National Centre for Physics, Quaid-I-Azam University, Islamabad, Pakistan

M. Ahmad, M.I. Asghar, H.R. Hoorani, S. Khalid, W.A. Khan, T. Khurshid, S. Qazi, M.A. Shah, M. Shoaib

Institute of Experimental Physics, Faculty of Physics, University of Warsaw, Warsaw, Poland

G. Brona, M. Cwiok, W. Dominik, K. Doroba, A. Kalinowski, M. Konecki, J. Krolikowski

Soltan Institute for Nuclear Studies, Warsaw, Poland

H. Bialkowska, B. Boimska, T. Frueboes, R. Gokieli, M. Górski, M. Kazana, K. Nawrocki,

K. Romanowska-Rybinska, M. Szleper, G. Wrochna, P. Zalewski

Laboratório de Instrumentação e Física Experimental de Partículas, Lisboa, Portugal

N. Almeida, P. Bargassa, A. David, P. Faccioli, P.G. Ferreira Parracho, M. Gallinaro, P. Musella, A. Nayak, J. Pela ${ }^{1}$, P.Q. Ribeiro, J. Seixas, J. Varela, P. Vischia

Joint Institute for Nuclear Research, Dubna, Russia

S. Afanasiev, I. Belotelov, P. Bunin, I. Golutvin, A. Kamenev, V. Karjavin, V. Konoplyanikov, G. Kozlov, A. Lanev, P. Moisenz, V. Palichik, V. Perelygin, M. Savina, S. Shmatov, V. Smirnov, A. Volodko, A. Zarubin

Petersburg Nuclear Physics Institute, Gatchina (St Petersburg), Russia

S. Evstyukhin, V. Golovtsov, Y. Ivanov, V. Kim, P. Levchenko, V. Murzin, V. Oreshkin, I. Smirnov, V. Sulimov, L. Uvarov, S. Vavilov, A. Vorobyev, An. Vorobyev

Institute for Nuclear Research, Moscow, Russia

Yu. Andreev, A. Dermenev, S. Gninenko, N. Golubev, M. Kirsanov, N. Krasnikov, V. Matveev, A. Pashenkov, A. Toropin, S. Troitsky

Institute for Theoretical and Experimental Physics, Moscow, Russia

V. Epshteyn, M. Erofeeva, V. Gavrilov, M. Kossov ${ }^{1}$, A. Krokhotin, N. Lychkovskaya, V. Popov, G. Safronov, S. Semenov, V. Stolin, E. Vlasov, A. Zhokin

Moscow State University, Moscow, Russia

A. Belyaev, E. Boos, M. Dubinin ${ }^{4}$, L. Dudko, A. Ershov, A. Gribushin, O. Kodolova, I. Lokhtin, A. Markina, S. Obraztsov, M. Perfilov, S. Petrushanko, L. Sarycheva ${ }^{\dagger}$, V. Savrin, A. Snigirev

P.N. Lebedev Physical Institute, Moscow, Russia

V. Andreev, M. Azarkin, I. Dremin, M. Kirakosyan, A. Leonidov, G. Mesyats, S.V. Rusakov, A. Vinogradov 
State Research Center of Russian Federation, Institute for High Energy Physics, Protvino, Russia

I. Azhgirey, I. Bayshev, S. Bitioukov, V. Grishin ${ }^{1}$, V. Kachanov, D. Konstantinov, A. Korablev, V. Krychkine, V. Petrov, R. Ryutin, A. Sobol, L. Tourtchanovitch, S. Troshin, N. Tyurin, A. Uzunian, A. Volkov

University of Belgrade, Faculty of Physics and Vinca Institute of Nuclear Sciences, Belgrade, Serbia

P. Adzic ${ }^{28}$, M. Djordjevic, M. Ekmedzic, D. Krpic ${ }^{28}$, J. Milosevic

Centro de Investigaciones Energéticas Medioambientales y Tecnológicas (CIEMAT), Madrid, Spain

M. Aguilar-Benitez, J. Alcaraz Maestre, P. Arce, C. Battilana, E. Calvo, M. Cerrada, M. Chamizo Llatas, N. Colino, B. De La Cruz, A. Delgado Peris, C. Diez Pardos, D. Domínguez Vázquez, C. Fernandez Bedoya, J.P. Fernández Ramos, A. Ferrando, J. Flix, M.C. Fouz, P. Garcia-Abia, O. Gonzalez Lopez, S. Goy Lopez, J.M. Hernandez, M.I. Josa, G. Merino, J. Puerta Pelayo, I. Redondo, L. Romero, J. Santaolalla, M.S. Soares, C. Willmott

Universidad Autónoma de Madrid, Madrid, Spain

C. Albajar, G. Codispoti, J.F. de Trocóniz

Universidad de Oviedo, Oviedo, Spain

J. Cuevas, J. Fernandez Menendez, S. Folgueras, I. Gonzalez Caballero, L. Lloret Iglesias, J. Piedra Gomez ${ }^{29}$, J.M. Vizan Garcia

Instituto de Física de Cantabria (IFCA), CSIC-Universidad de Cantabria, Santander, Spain

J.A. Brochero Cifuentes, I.J. Cabrillo, A. Calderon, S.H. Chuang, J. Duarte Campderros, M. Felcini ${ }^{30}$, M. Fernandez, G. Gomez, J. Gonzalez Sanchez, C. Jorda, P. Lobelle Pardo, A. Lopez Virto, J. Marco, R. Marco, C. Martinez Rivero, F. Matorras, F.J. Munoz Sanchez, T. Rodrigo, A.Y. Rodríguez-Marrero, A. Ruiz-Jimeno, L. Scodellaro, M. Sobron Sanudo, I. Vila, R. Vilar Cortabitarte

CERN, European Organization for Nuclear Research, Geneva, Switzerland

D. Abbaneo, E. Auffray, G. Auzinger, P. Baillon, A.H. Ball, D. Barney, C. Bernet ${ }^{5}$, W. Bialas, G. Bianchi, P. Bloch, A. Bocci, H. Breuker, K. Bunkowski, T. Camporesi, G. Cerminara, T. Christiansen, J.A. Coarasa Perez, B. Curé, D. D'Enterria, A. De Roeck, S. Di Guida, M. Dobson, N. Dupont-Sagorin, A. Elliott-Peisert, B. Frisch, W. Funk, A. Gaddi, G. Georgiou, H. Gerwig, M. Giffels, D. Gigi, K. Gill, D. Giordano, M. Giunta, F. Glege, R. Gomez-Reino Garrido, P. Govoni, S. Gowdy, R. Guida, L. Guiducci, M. Hansen, P. Harris, C. Hartl, J. Harvey, B. Hegner, A. Hinzmann, H.F. Hoffmann, V. Innocente, P. Janot, K. Kaadze, E. Karavakis, K. Kousouris, P. Lecoq, P. Lenzi, C. Lourenço, T. Mäki, M. Malberti, L. Malgeri, M. Mannelli, L. Masetti, G. Mavromanolakis, F. Meijers, S. Mersi, E. Meschi, R. Moser, M.U. Mozer, M. Mulders, E. Nesvold, M. Nguyen, T. Orimoto, L. Orsini, E. Palencia Cortezon, E. Perez, A. Petrilli, A. Pfeiffer, M. Pierini, 
M. Pimiä, D. Piparo, G. Polese, L. Quertenmont, A. Racz, W. Reece, J. Rodrigues Antunes, G. Rolandi ${ }^{31}$, T. Rommerskirchen, C. Rovelli ${ }^{32}$, M. Rovere, H. Sakulin, F. Santanastasio, C. Schäfer, C. Schwick, I. Segoni, A. Sharma, P. Siegrist, P. Silva, M. Simon, P. Sphicas ${ }^{33}$, D. Spiga, M. Spiropulu ${ }^{4}$, M. Stoye, A. Tsirou, G.I. Veres ${ }^{16}$, P. Vichoudis, H.K. Wöhri, S.D. Worm ${ }^{34}$, W.D. Zeuner

\section{Paul Scherrer Institut, Villigen, Switzerland}

W. Bertl, K. Deiters, W. Erdmann, K. Gabathuler, R. Horisberger, Q. Ingram, H.C. Kaestli, S. König, D. Kotlinski, U. Langenegger, F. Meier, D. Renker, T. Rohe, J. Sibille ${ }^{35}$

\section{Institute for Particle Physics, ETH Zurich, Zurich, Switzerland}

L. Bäni, P. Bortignon, M.A. Buchmann, B. Casal, N. Chanon, Z. Chen, A. Deisher, G. Dissertori, M. Dittmar, M. Dünser, J. Eugster, K. Freudenreich, C. Grab, P. Lecomte, W. Lustermann, P. Martinez Ruiz del Arbol, N. Mohr, F. Moortgat, C. Nägeli ${ }^{36}$, P. Nef, F. Nessi-Tedaldi, L. Pape, F. Pauss, M. Peruzzi, F.J. Ronga, M. Rossini, L. Sala, A.K. Sanchez, M.-C. Sawley, A. Starodumov ${ }^{37}$, B. Stieger, M. Takahashi, L. Tauscher ${ }^{\dagger}$, A. Thea, K. Theofilatos, D. Treille, C. Urscheler, R. Wallny, H.A. Weber, L. Wehrli, J. Weng

\section{Universität Zürich, Zurich, Switzerland}

E. Aguilo, C. Amsler, V. Chiochia, S. De Visscher, C. Favaro, M. Ivova Rikova, B. Millan Mejias, P. Otiougova, P. Robmann, H. Snoek, M. Verzetti

\section{National Central University, Chung-Li, Taiwan}

Y.H. Chang, K.H. Chen, C.M. Kuo, S.W. Li, W. Lin, Z.K. Liu, Y.J. Lu, D. Mekterovic, R. Volpe, S.S. Yu

\section{National Taiwan University (NTU), Taipei, Taiwan}

P. Bartalini, P. Chang, Y.H. Chang, Y.W. Chang, Y. Chao, K.F. Chen, C. Dietz, U. Grundler, W.-S. Hou, Y. Hsiung, K.Y. Kao, Y.J. Lei, R.-S. Lu, D. Majumder, E. Petrakou, X. Shi, J.G. Shiu, Y.M. Tzeng, M. Wang

\section{Cukurova University, Adana, Turkey}

A. Adiguzel, M.N. Bakirci ${ }^{38}$, S. Cerci ${ }^{39}$, C. Dozen, I. Dumanoglu, E. Eskut, S. Girgis, G. Gokbulut, I. Hos, E.E. Kangal, G. Karapinar, A. Kayis Topaksu, G. Onengut, K. Ozdemir, S. Ozturk ${ }^{40}$, A. Polatoz, K. Sogut ${ }^{41}$, D. Sunar Cerci ${ }^{39}$, B. Tali ${ }^{39}$, H. Topakli ${ }^{38}$, D. Uzun, L.N. Vergili, M. Vergili

\section{Middle East Technical University, Physics Department, Ankara, Turkey}

I.V. Akin, T. Aliev, B. Bilin, S. Bilmis, M. Deniz, H. Gamsizkan, A.M. Guler, K. Ocalan, A. Ozpineci, M. Serin, R. Sever, U.E. Surat, M. Yalvac, E. Yildirim, M. Zeyrek

\section{Bogazici University, Istanbul, Turkey}

M. Deliomeroglu, E. Gülmez, B. Isildak, M. Kaya ${ }^{42}$, O. Kaya ${ }^{42}$, S. Ozkorucuklu ${ }^{43}$, N. Sonmez ${ }^{44}$ 
National Scientific Center, Kharkov Institute of Physics and Technology, Kharkov, Ukraine

L. Levchuk

\section{University of Bristol, Bristol, United Kingdom}

F. Bostock, J.J. Brooke, E. Clement, D. Cussans, H. Flacher, R. Frazier, J. Goldstein, M. Grimes, G.P. Heath, H.F. Heath, L. Kreczko, S. Metson, D.M. Newbold ${ }^{34}$, K. Nirunpong, A. Poll, S. Senkin, V.J. Smith, T. Williams

\section{Rutherford Appleton Laboratory, Didcot, United Kingdom}

L. Basso ${ }^{45}$, K.W. Bell, A. Belyaev ${ }^{45}$, C. Brew, R.M. Brown, D.J.A. Cockerill, J.A. Coughlan, K. Harder, S. Harper, J. Jackson, B.W. Kennedy, E. Olaiya, D. Petyt, B.C. RadburnSmith, C.H. Shepherd-Themistocleous, I.R. Tomalin, W.J. Womersley

\section{Imperial College, London, United Kingdom}

R. Bainbridge, G. Ball, R. Beuselinck, O. Buchmuller, D. Colling, N. Cripps, M. Cutajar, P. Dauncey, G. Davies, M. Della Negra, W. Ferguson, J. Fulcher, D. Futyan, A. Gilbert, A. Guneratne Bryer, G. Hall, Z. Hatherell, J. Hays, G. Iles, M. Jarvis, G. Karapostoli, L. Lyons, A.-M. Magnan, J. Marrouche, B. Mathias, R. Nandi, J. Nash, A. Nikitenko ${ }^{37}$, A. Papageorgiou, M. Pesaresi, K. Petridis, M. Pioppi ${ }^{46}$, D.M. Raymond, S. Rogerson, N. Rompotis, A. Rose, M.J. Ryan, C. Seez, A. Sparrow, A. Tapper, S. Tourneur, M. Vazquez Acosta, T. Virdee, S. Wakefield, N. Wardle, D. Wardrope, T. Whyntie

\section{Brunel University, Uxbridge, United Kingdom}

M. Barrett, M. Chadwick, J.E. Cole, P.R. Hobson, A. Khan, P. Kyberd, D. Leslie, W. Martin, I.D. Reid, P. Symonds, L. Teodorescu, M. Turner

\section{Baylor University, Waco, USA}

K. Hatakeyama, H. Liu, T. Scarborough

The University of Alabama, Tuscaloosa, USA

C. Henderson

\section{Boston University, Boston, USA}

A. Avetisyan, T. Bose, E. Carrera Jarrin, C. Fantasia, A. Heister, J. St. John, P. Lawson, D. Lazic, J. Rohlf, D. Sperka, L. Sulak

\section{Brown University, Providence, USA}

S. Bhattacharya, D. Cutts, A. Ferapontov, U. Heintz, S. Jabeen, G. Kukartsev, G. Landsberg, M. Luk, M. Narain, D. Nguyen, M. Segala, T. Sinthuprasith, T. Speer, K.V. Tsang

\section{University of California, Davis, Davis, USA}

R. Breedon, G. Breto, M. Calderon De La Barca Sanchez, M. Caulfield, S. Chauhan, M. Chertok, J. Conway, R. Conway, P.T. Cox, J. Dolen, R. Erbacher, M. Gardner, R. Houtz, W. Ko, A. Kopecky, R. Lander, O. Mall, T. Miceli, R. Nelson, D. Pellett, J. Robles, B. Rutherford, M. Searle, J. Smith, M. Squires, M. Tripathi, R. Vasquez Sierra 
University of California, Los Angeles, Los Angeles, USA

V. Andreev, K. Arisaka, D. Cline, R. Cousins, J. Duris, S. Erhan, P. Everaerts, C. Farrell, J. Hauser, M. Ignatenko, C. Jarvis, C. Plager, G. Rakness, P. Schlein ${ }^{\dagger}$, J. Tucker, V. Valuev, M. Weber

University of California, Riverside, Riverside, USA

J. Babb, R. Clare, J. Ellison, J.W. Gary, F. Giordano, G. Hanson, G.Y. Jeng, H. Liu, O.R. Long, A. Luthra, H. Nguyen, S. Paramesvaran, J. Sturdy, S. Sumowidagdo, R. Wilken, S. Wimpenny

\section{University of California, San Diego, La Jolla, USA}

W. Andrews, J.G. Branson, G.B. Cerati, S. Cittolin, D. Evans, F. Golf, A. Holzner, R. Kelley, M. Lebourgeois, J. Letts, I. Macneill, B. Mangano, S. Padhi, C. Palmer, G. Petrucciani, H. Pi, M. Pieri, R. Ranieri, M. Sani, I. Sfiligoi, V. Sharma, S. Simon, E. Sudano, M. Tadel, Y. Tu, A. Vartak, S. Wasserbaech ${ }^{47}$, F. Würthwein, A. Yagil, J. Yoo

\section{University of California, Santa Barbara, Santa Barbara, USA}

D. Barge, R. Bellan, C. Campagnari, M. D'Alfonso, T. Danielson, K. Flowers, P. Geffert, J. Incandela, C. Justus, P. Kalavase, S.A. Koay, D. Kovalskyi ${ }^{1}$, V. Krutelyov, S. Lowette, N. Mccoll, V. Pavlunin, F. Rebassoo, J. Ribnik, J. Richman, R. Rossin, D. Stuart, W. To, J.R. Vlimant, C. West

\section{California Institute of Technology, Pasadena, USA}

A. Apresyan, A. Bornheim, J. Bunn, Y. Chen, E. Di Marco, J. Duarte, M. Gataullin, Y. Ma, A. Mott, H.B. Newman, C. Rogan, V. Timciuc, P. Traczyk, J. Veverka, R. Wilkinson, Y. Yang, R.Y. Zhu

\section{Carnegie Mellon University, Pittsburgh, USA}

B. Akgun, R. Carroll, T. Ferguson, Y. Iiyama, D.W. Jang, S.Y. Jun, Y.F. Liu, M. Paulini, J. Russ, H. Vogel, I. Vorobiev

University of Colorado at Boulder, Boulder, USA

J.P. Cumalat, M.E. Dinardo, B.R. Drell, C.J. Edelmaier, W.T. Ford, A. Gaz, B. Heyburn, E. Luiggi Lopez, U. Nauenberg, J.G. Smith, K. Stenson, K.A. Ulmer, S.R. Wagner, S.L. Zang

\section{Cornell University, Ithaca, USA}

L. Agostino, J. Alexander, A. Chatterjee, N. Eggert, L.K. Gibbons, B. Heltsley, W. Hopkins, A. Khukhunaishvili, B. Kreis, N. Mirman, G. Nicolas Kaufman, J.R. Patterson, A. Ryd, E. Salvati, W. Sun, W.D. Teo, J. Thom, J. Thompson, J. Vaughan, Y. Weng, L. Winstrom, P. Wittich

\section{Fairfield University, Fairfield, USA}

A. Biselli, D. Winn

\section{Fermi National Accelerator Laboratory, Batavia, USA}

S. Abdullin, M. Albrow, J. Anderson, G. Apollinari, M. Atac, J.A. Bakken, L.A.T. Bauerdick, A. Beretvas, J. Berryhill, P.C. Bhat, I. Bloch, K. Burkett, J.N. Butler, 
V. Chetluru, H.W.K. Cheung, F. Chlebana, S. Cihangir, W. Cooper, D.P. Eartly, V.D. Elvira, S. Esen, I. Fisk, J. Freeman, Y. Gao, E. Gottschalk, D. Green, O. Gutsche, J. Hanlon, R.M. Harris, J. Hirschauer, B. Hooberman, H. Jensen, S. Jindariani, M. Johnson, U. Joshi, B. Klima, S. Kunori, S. Kwan, C. Leonidopoulos, D. Lincoln, R. Lipton, J. Lykken, K. Maeshima, J.M. Marraffino, S. Maruyama, D. Mason, P. McBride, T. Miao, K. Mishra, S. Mrenna, Y. Musienko ${ }^{48}$, C. Newman-Holmes, V. O’Dell, J. Pivarski, R. Pordes, O. Prokofyev, T. Schwarz, E. Sexton-Kennedy, S. Sharma, W.J. Spalding, L. Spiegel, P. Tan, L. Taylor, S. Tkaczyk, L. Uplegger, E.W. Vaandering, R. Vidal, J. Whitmore, W. Wu, F. Yang, F. Yumiceva, J.C. Yun

\section{University of Florida, Gainesville, USA}

D. Acosta, P. Avery, D. Bourilkov, M. Chen, S. Das, M. De Gruttola, G.P. Di Giovanni, D. Dobur, A. Drozdetskiy, R.D. Field, M. Fisher, Y. Fu, I.K. Furic, J. Gartner, S. Goldberg, J. Hugon, B. Kim, J. Konigsberg, A. Korytov, A. Kropivnitskaya, T. Kypreos, J.F. Low, K. Matchev, P. Milenovic ${ }^{49}$, G. Mitselmakher, L. Muniz, R. Remington, A. Rinkevicius, M. Schmitt, B. Scurlock, P. Sellers, N. Skhirtladze, M. Snowball, D. Wang, J. Yelton, M. Zakaria

\section{Florida International University, Miami, USA}

V. Gaultney, L.M. Lebolo, S. Linn, P. Markowitz, G. Martinez, J.L. Rodriguez

\section{Florida State University, Tallahassee, USA}

T. Adams, A. Askew, J. Bochenek, J. Chen, B. Diamond, S.V. Gleyzer, J. Haas, S. Hagopian, V. Hagopian, M. Jenkins, K.F. Johnson, H. Prosper, S. Sekmen, V. Veeraraghavan, M. Weinberg

\section{Florida Institute of Technology, Melbourne, USA}

M.M. Baarmand, B. Dorney, M. Hohlmann, H. Kalakhety, I. Vodopiyanov

\section{University of Illinois at Chicago (UIC), Chicago, USA}

M.R. Adams, I.M. Anghel, L. Apanasevich, Y. Bai, V.E. Bazterra, R.R. Betts, J. Callner, R. Cavanaugh, C. Dragoiu, L. Gauthier, C.E. Gerber, D.J. Hofman, S. Khalatyan, G.J. Kunde ${ }^{50}$, F. Lacroix, M. Malek, C. O'Brien, C. Silkworth, C. Silvestre, D. Strom, N. Varelas

\section{The University of Iowa, Iowa City, USA}

U. Akgun, E.A. Albayrak, B. Bilki ${ }^{51}$, W. Clarida, F. Duru, S. Griffiths, C.K. Lae, E. McCliment, J.-P. Merlo, H. Mermerkaya ${ }^{52}$, A. Mestvirishvili, A. Moeller, J. Nachtman, C.R. Newsom, E. Norbeck, J. Olson, Y. Onel, F. Ozok, S. Sen, E. Tiras, J. Wetzel, T. Yetkin, K. Yi

\section{Johns Hopkins University, Baltimore, USA}

B.A. Barnett, B. Blumenfeld, S. Bolognesi, A. Bonato, D. Fehling, G. Giurgiu, A.V. Gritsan, Z.J. Guo, G. Hu, P. Maksimovic, S. Rappoccio, M. Swartz, N.V. Tran, A. Whitbeck

\section{The University of Kansas, Lawrence, USA}

P. Baringer, A. Bean, G. Benelli, O. Grachov, R.P. Kenny Iii, M. Murray, D. Noonan, S. Sanders, R. Stringer, G. Tinti, J.S. Wood, V. Zhukova 
Kansas State University, Manhattan, USA

A.F. Barfuss, T. Bolton, I. Chakaberia, A. Ivanov, S. Khalil, M. Makouski, Y. Maravin, S. Shrestha, I. Svintradze

Lawrence Livermore National Laboratory, Livermore, USA

J. Gronberg, D. Lange, D. Wright

University of Maryland, College Park, USA

A. Baden, M. Boutemeur, B. Calvert, S.C. Eno, J.A. Gomez, N.J. Hadley, R.G. Kellogg, M. Kirn, T. Kolberg, Y. Lu, M. Marionneau, A.C. Mignerey, A. Peterman, K. Rossato, P. Rumerio, A. Skuja, J. Temple, M.B. Tonjes, S.C. Tonwar, E. Twedt

Massachusetts Institute of Technology, Cambridge, USA

B. Alver, G. Bauer, J. Bendavid, W. Busza, E. Butz, I.A. Cali, M. Chan, V. Dutta, G. Gomez Ceballos, M. Goncharov, K.A. Hahn, Y. Kim, M. Klute, Y.-J. Lee, W. Li, P.D. Luckey, T. Ma, S. Nahn, C. Paus, D. Ralph, C. Roland, G. Roland, M. Rudolph, G.S.F. Stephans, F. Stöckli, K. Sumorok, K. Sung, D. Velicanu, E.A. Wenger, R. Wolf, B. Wyslouch, S. Xie, M. Yang, Y. Yilmaz, A.S. Yoon, M. Zanetti

University of Minnesota, Minneapolis, USA

S.I. Cooper, P. Cushman, B. Dahmes, A. De Benedetti, G. Franzoni, A. Gude, J. Haupt, S.C. Kao, K. Klapoetke, Y. Kubota, J. Mans, N. Pastika, V. Rekovic, R. Rusack, M. Sasseville, A. Singovsky, N. Tambe, J. Turkewitz

University of Mississippi, University, USA

L.M. Cremaldi, R. Godang, R. Kroeger, L. Perera, R. Rahmat, D.A. Sanders, D. Summers

\section{University of Nebraska-Lincoln, Lincoln, USA}

E. Avdeeva, K. Bloom, S. Bose, J. Butt, D.R. Claes, A. Dominguez, M. Eads, P. Jindal, J. Keller, I. Kravchenko, J. Lazo-Flores, H. Malbouisson, S. Malik, G.R. Snow

State University of New York at Buffalo, Buffalo, USA

U. Baur, A. Godshalk, I. Iashvili, S. Jain, A. Kharchilava, A. Kumar, S.P. Shipkowski, K. Smith, Z. Wan

Northeastern University, Boston, USA

G. Alverson, E. Barberis, D. Baumgartel, M. Chasco, D. Trocino, D. Wood, J. Zhang

Northwestern University, Evanston, USA

A. Anastassov, A. Kubik, N. Mucia, N. Odell, R.A. Ofierzynski, B. Pollack, A. Pozdnyakov, M. Schmitt, S. Stoynev, M. Velasco, S. Won

University of Notre Dame, Notre Dame, USA

L. Antonelli, D. Berry, A. Brinkerhoff, M. Hildreth, C. Jessop, D.J. Karmgard, J. Kolb, K. Lannon, W. Luo, S. Lynch, N. Marinelli, D.M. Morse, T. Pearson, R. Ruchti, J. Slaunwhite, N. Valls, M. Wayne, M. Wolf, J. Ziegler 


\section{The Ohio State University, Columbus, USA}

B. Bylsma, L.S. Durkin, C. Hill, P. Killewald, K. Kotov, T.Y. Ling, D. Puigh, M. Rodenburg, C. Vuosalo, G. Williams

\section{Princeton University, Princeton, USA}

N. Adam, E. Berry, P. Elmer, D. Gerbaudo, V. Halyo, P. Hebda, J. Hegeman, A. Hunt, E. Laird, D. Lopes Pegna, P. Lujan, D. Marlow, T. Medvedeva, M. Mooney, J. Olsen, P. Piroué, X. Quan, A. Raval, H. Saka, D. Stickland, C. Tully, J.S. Werner, A. Zuranski

\section{University of Puerto Rico, Mayaguez, USA}

J.G. Acosta, X.T. Huang, A. Lopez, H. Mendez, S. Oliveros, J.E. Ramirez Vargas, A. Zatserklyaniy

\section{Purdue University, West Lafayette, USA}

E. Alagoz, V.E. Barnes, D. Benedetti, G. Bolla, D. Bortoletto, M. De Mattia, A. Everett, L. Gutay, Z. Hu, M. Jones, O. Koybasi, M. Kress, A.T. Laasanen, N. Leonardo, V. Maroussov, P. Merkel, D.H. Miller, N. Neumeister, I. Shipsey, D. Silvers, A. Svyatkovskiy, M. Vidal Marono, H.D. Yoo, J. Zablocki, Y. Zheng

Purdue University Calumet, Hammond, USA

S. Guragain, N. Parashar

\section{Rice University, Houston, USA}

A. Adair, C. Boulahouache, V. Cuplov, K.M. Ecklund, F.J.M. Geurts, B.P. Padley, R. Redjimi, J. Roberts, J. Zabel

University of Rochester, Rochester, USA

B. Betchart, A. Bodek, Y.S. Chung, R. Covarelli, P. de Barbaro, R. Demina, Y. Eshaq, A. Garcia-Bellido, P. Goldenzweig, Y. Gotra, J. Han, A. Harel, D.C. Miner, G. Petrillo, W. Sakumoto, D. Vishnevskiy, M. Zielinski

\section{The Rockefeller University, New York, USA}

A. Bhatti, R. Ciesielski, L. Demortier, K. Goulianos, G. Lungu, S. Malik, C. Mesropian

\section{Rutgers, the State University of New Jersey, Piscataway, USA}

S. Arora, O. Atramentov, A. Barker, J.P. Chou, C. Contreras-Campana, E. ContrerasCampana, D. Duggan, D. Ferencek, Y. Gershtein, R. Gray, E. Halkiadakis, D. Hidas, D. Hits, A. Lath, S. Panwalkar, M. Park, R. Patel, A. Richards, K. Rose, S. Salur, S. Schnetzer, C. Seitz, S. Somalwar, R. Stone, S. Thomas

\section{University of Tennessee, Knoxville, USA}

G. Cerizza, M. Hollingsworth, S. Spanier, Z.C. Yang, A. York

\section{Texas A\&M University, College Station, USA}

R. Eusebi, W. Flanagan, J. Gilmore, T. Kamon ${ }^{53}$, V. Khotilovich, R. Montalvo, I. Osipenkov, Y. Pakhotin, A. Perloff, J. Roe, A. Safonov, T. Sakuma, S. Sengupta, I. Suarez, A. Tatarinov, D. Toback 


\title{
Texas Tech University, Lubbock, USA
}

N. Akchurin, C. Bardak, J. Damgov, P.R. Dudero, C. Jeong, K. Kovitanggoon, S.W. Lee, T. Libeiro, P. Mane, Y. Roh, A. Sill, I. Volobouev, R. Wigmans

\section{Vanderbilt University, Nashville, USA}

E. Appelt, E. Brownson, D. Engh, C. Florez, W. Gabella, A. Gurrola, M. Issah, W. Johns, P. Kurt, C. Maguire, A. Melo, P. Sheldon, B. Snook, S. Tuo, J. Velkovska

\section{University of Virginia, Charlottesville, USA}

M.W. Arenton, M. Balazs, S. Boutle, S. Conetti, B. Cox, B. Francis, S. Goadhouse, J. Goodell, R. Hirosky, A. Ledovskoy, C. Lin, C. Neu, J. Wood, R. Yohay

\section{Wayne State University, Detroit, USA}

S. Gollapinni, R. Harr, P.E. Karchin, C. Kottachchi Kankanamge Don, P. Lamichhane, M. Mattson, C. Milstène, A. Sakharov

\section{University of Wisconsin, Madison, USA}

M. Anderson, M. Bachtis, D. Belknap, J.N. Bellinger, J. Bernardini, L. Borrello, D. Carlsmith, M. Cepeda, S. Dasu, J. Efron, E. Friis, L. Gray, K.S. Grogg, M. Grothe, R. HallWilton, M. Herndon, A. Hervé, P. Klabbers, J. Klukas, A. Lanaro, C. Lazaridis, J. Leonard, R. Loveless, A. Mohapatra, I. Ojalvo, G.A. Pierro, I. Ross, A. Savin, W.H. Smith, J. Swanson

\author{
$\dagger$ : Deceased \\ 1: Also at CERN, European Organization for Nuclear Research, Geneva, Switzerland \\ 2: Also at National Institute of Chemical Physics and Biophysics, Tallinn, Estonia \\ 3: Also at Universidade Federal do ABC, Santo Andre, Brazil \\ 4: Also at California Institute of Technology, Pasadena, USA \\ 5: Also at Laboratoire Leprince-Ringuet, Ecole Polytechnique, IN2P3-CNRS, Palaiseau, France \\ 6: Also at Suez Canal University, Suez, Egypt \\ 7: Also at Cairo University, Cairo, Egypt \\ 8: Also at British University, Cairo, Egypt \\ 9: Also at Fayoum University, El-Fayoum, Egypt \\ 10: Now at Ain Shams University, Cairo, Egypt \\ 11: Also at Soltan Institute for Nuclear Studies, Warsaw, Poland \\ 12: Also at Université de Haute-Alsace, Mulhouse, France \\ 13: Also at Moscow State University, Moscow, Russia \\ 14: Also at Brandenburg University of Technology, Cottbus, Germany \\ 15: Also at Institute of Nuclear Research ATOMKI, Debrecen, Hungary \\ 16: Also at Eötvös Loránd University, Budapest, Hungary \\ 17: Also at Tata Institute of Fundamental Research - HECR, Mumbai, India \\ 18: Now at King Abdulaziz University, Jeddah, Saudi Arabia \\ 19: Also at University of Visva-Bharati, Santiniketan, India \\ 20: Also at Sharif University of Technology, Tehran, Iran \\ 21: Also at Isfahan University of Technology, Isfahan, Iran \\ 22: Also at Shiraz University, Shiraz, Iran \\ 23: Also at Plasma Physics Research Center, Science and Research Branch, Islamic Azad \\ University, Teheran, Iran
}


24: Also at Facoltà Ingegneria Università di Roma, Roma, Italy

25: Also at Università della Basilicata, Potenza, Italy

26: Also at Laboratori Nazionali di Legnaro dell' INFN, Legnaro, Italy

27: Also at Università degli studi di Siena, Siena, Italy

28: Also at Faculty of Physics of University of Belgrade, Belgrade, Serbia

29: Also at University of Florida, Gainesville, USA

30: Also at University of California, Los Angeles, Los Angeles, USA

31: Also at Scuola Normale e Sezione dell' INFN, Pisa, Italy

32: Also at INFN Sezione di Roma; Università di Roma "La Sapienza", Roma, Italy

33: Also at University of Athens, Athens, Greece

34: Also at Rutherford Appleton Laboratory, Didcot, United Kingdom

35: Also at The University of Kansas, Lawrence, USA

36: Also at Paul Scherrer Institut, Villigen, Switzerland

37: Also at Institute for Theoretical and Experimental Physics, Moscow, Russia

38: Also at Gaziosmanpasa University, Tokat, Turkey

39: Also at Adiyaman University, Adiyaman, Turkey

40: Also at The University of Iowa, Iowa City, USA

41: Also at Mersin University, Mersin, Turkey

42: Also at Kafkas University, Kars, Turkey

43: Also at Suleyman Demirel University, Isparta, Turkey

44: Also at Ege University, Izmir, Turkey

45: Also at School of Physics and Astronomy, University of Southampton, Southampton, United Kingdom

46: Also at INFN Sezione di Perugia; Università di Perugia, Perugia, Italy

47: Also at Utah Valley University, Orem, USA

48: Also at Institute for Nuclear Research, Moscow, Russia

49: Also at University of Belgrade, Faculty of Physics and Vinca Institute of Nuclear Sciences, Belgrade, Serbia

50: Also at Los Alamos National Laboratory, Los Alamos, USA

51: Also at Argonne National Laboratory, Argonne, USA

52: Also at Erzincan University, Erzincan, Turkey

53: Also at Kyungpook National University, Daegu, Korea 